\title{
Pricing and Risk Management with Stochastic Volatility Using Importance Sampling
}

Link to publication record in Manchester Research Explorer

\section{Citation for published version (APA):}

Stilger, P. S., Acomb, S., \& Poon, S-H. (2012). Pricing and Risk Management with Stochastic Volatility Using Importance Sampling. (Social Science Research Network; No. 2168415).

\section{Citing this paper}

Please note that where the full-text provided on Manchester Research Explorer is the Author Accepted Manuscript or Proof version this may differ from the final Published version. If citing, it is advised that you check and use the publisher's definitive version.

\section{General rights}

Copyright and moral rights for the publications made accessible in the Research Explorer are retained by the authors and/or other copyright owners and it is a condition of accessing publications that users recognise and abide by the legal requirements associated with these rights.

\section{Takedown policy}

If you believe that this document breaches copyright please refer to the University of Manchester's Takedown Procedures [http://man.ac.uk/04Y6Bo] or contact uml.scholarlycommunications@manchester.ac.uk providing relevant details, so we can investigate your claim.

\section{OPEN ACCESS}




\title{
Pricing and Risk Management with Stochastic Volatility Using Importance Sampling
}

\author{
Przemyslaw Stan Stilger, Simon Acomb and Ser-Huang Poon*
}

March 20, 2014

\begin{abstract}
In this paper, we apply importance sampling to Heston's stochastic volatility model and Bates's stochastic volatility model with jumps. We propose an effective numerical scheme that dramatically improves the speed of importance sampling. We show how the Greeks can be computed using the Likelihood Ratio Method based on characteristic function, and how combining it with importance sampling leads to a significant variance reduction for the Greeks.
\end{abstract} All results are illustrated using European and barrier options.

Keywords: Importance sampling; Simulation; Stochastic volatility

*Przemyslaw Stan Stilger (przemyslaw.stilger@postgrad.mbs.ac.uk), Simon Acomb and Ser-Huang Poon (serhuang.poon@mbs.ac.uk) are at Manchester Business School, Crawford House, University of Manchester, Oxford Road, Manchester, M13 9PL, United Kingdom. We thank Eberhard Mayerhofer for helpful discussions and comments. Stilger gratefully acknowledges financial support from the ESRC. 


\section{Introduction}

This paper extends the application of importance sampling to derivative pricing with price dynamics that have stochastic volatility and jumps. Our contributions are as follows. First, we apply the importance sampling scheme introduced in Fouque and Tullie (2002) to price derivatives under the Heston and the Bates models. To accommodate the jumps in the Bates model, we add a modification so that the effect of jump is captured by the change in volatility of the stock price. Consequently, it is possible to apply importance sampling to the Bates model. Next, we introduce an effective numerical scheme based on a 2-dimensinal lookup table that dramatically improves the speed of importance sampling scheme. Finally, we demonstrate how the Likelihood Ratio Method for calculating the Greeks can be made more efficient by importance sampling.

It is well known that the error rate in Monte Carlo estimator decreases with $\sqrt{n}$ where $n$ is the number of sample paths. This has an important implication on the speed of the simulation. For example, to reduce the error by a factor of 10, Monte Carlo simulation requires 100 times more samples and thus 100 times more computational effort. Many techniques have been suggested to improve the efficiency of Monte Carlo simulation including: antithetic variates, control variate, importance sampling, and stratified sampling. Here, we focus on the variance reduction through importance sampling. The main idea behind importance sampling is to focus the simulation effort on the event of major concern. In option pricing, this amounts to simulating only sample paths that will lead to a non-zero payoff. This is done by changing the drift of the simulated sample paths and then rescaling the payoff by the likelihood ratio to obtain the actual probability. From the mathematical point of view, this likelihood ratio is the Radon-Nikodym derivative of the original probability measure with respect to the importance sampling measure. It is important to note here that the variance reduction achieved through importance sampling critically depends on the change of drift.

The rest of the paper is organized as follows. In Section 2, we discuss how this paper is set in context with the previous literature. In Section 3, we follow Fouque and Tullie (2002) to derive the optimal change of drift for the Heston model. Next, we recall the approximations to European option prices obtained using small noise and fast mean-reversion expansions. Small noise expansion was introduced in Fournie, Lebuchoux and Touzi (1997) and is obtained by regular perturbation of the pricing partial differential equation. Fast mean-reversion expansion was introduced in Fouque and Tullie (2002) and is obtained by singular perturbation of the pricing partial differential equa- 
tion. We then use these expansions together with the optimal change of drift and apply them to European and barrier options. This results in two approximations to the optimal change of drift. The first approximation to the optimal change of drift is based on the small noise expansion, whereas the second one is based on the fast mean-reversion expansion. Finally, we discuss how to improve the speed of both small noise and fast mean-reversion importance sampling schemes by using a 2-dimensinal lookup table. In Section 4, we extend the results in Section 3 and derive the optimal change of measure for the Bates model. In Section 5, we show how the Greeks can be computed using the Likelihood Ratio Method. We then improve the computational efficiency by using the importance sampling schemes, and demonstrate their performance using European and barrier options in both Heston and Bates models. Finally, Section 6 concludes the paper. Throughout this paper we use a European call and a down-and-out put as examples.

\section{Literature Review}

Importance sampling is a popular variance reduction technique. Much research has been devoted to importance sampling and the choice of drift that maximizes the variance reduction. One of the areas where importance sampling finds fruitful applications is credit risk, because it is able to capture the rare but large losses that are very important in credit risk. For example, Merino and Nyfeler (2004) apply importance sampling in the estimation of individual risk contributions of the obligors to the expected shortfall of the credit portfolio. Glasserman (2005) also uses importance sampling to estimate marginal risk contributions to the portfolio expected shortfall and value-atrisk. Glasserman (2005) notes that individual risk contributions can be thought of as marginal risk contributions and calculated as expected losses conditioned on a tail event. Since calculating these conditional expectations requires the simulation of rare events, importance sampling focuses the simulation effort on these rare events thereby dramatically increases the convergence of the Monte Carlo estimator.

In a copula literature, Glasserman and Li (2005) introduce asymptotically optimal changes of measure for the estimation of credit risk with the Gaussian copula. Glasserman, Kang and Shahabuddin (2008) extend the work of Glasserman and Li (2005) to the multifactor Gaussian copula and Kang and Shahabuddin (2005) to the t-copula. Huang et. al. (2010) demonstrate the application of importance sampling to estimate conditional value-at-risk in the Gaussian copula model. Sak and Hörmann (2012) use importance sampling in the same model to estimate the probability of large losses and expected shortfall. Chan and Kroese (2010) utilize importance sampling to estimate the probability of large losses in the t-copula model. Grudke (2009) shows 
the benefit of using importance sampling for calculating economic capital.

In credit derivatives, Joshi and Kainth (2004) introduce an importance sampling procedure for the pricing of n-th to default swaps. Chen and Glasserman (2008) improve their method by changing the default probabilities and use it for the pricing of basket default swaps. Joshi (2004) shows that importance sampling can be applied to the pricing of single tranche CDOs. In case of default swaps and CDOs the advantage of importance sampling is the concentration of the simulation effort in regions where defaults are most likely.

In option pricing, Vázquez-Abad and Dufresne (1998) introduce a variance reduction method that combines importance sampling and control variate for Asian options in the Black-Scholes model. Glasserman et al. (1999a) propose a variance reduction method for path-dependent options that is based on importance sampling with deterministic change of drift and stratified sampling. They show that the variance of the Monte Carlo estimator can be approximated using the Laplace method for integrals. Based on that, they identify the asymptotically optimal change of drift as a solution to a deterministic optimization problem in discrete time. In a subsequent paper, Glasserman et al. (1999b) apply this method in the Heath-Jarrow-Morton framework. Étoré and Jourdain (2010) improve the method of Glasserman et al. (1999a) by using stratified sampling with adaptive allocation. Guasoni and Robertson (2008) generalize the importance sampling result of Glasserman et al. (1999a) to continuous time. The asymptotically optimal change of drift is defined as a solution to a variational problem that reduces to an Euler-Lagrange equation. Vázquez-Abad and Dufresne (1998), Su and Fu (1999), as well as Arouna (2004) use stochastic approximation methods to determine the optimal change of drift. In the least squares importance sampling of Caprotti (2008), the change of drift is formulated in terms of least squares minimization problem. As shown in Caprotti (2007), least squares importance sampling can be also used for LIBOR market model. The common feature of these studies is that the optimal change of drift is obtained by solving an optimization problem.

Fouque and Tullie (2002) develop a variance reduction method based on importance sampling with a stochastic change of drift for pricing options under stochastic volatility. The change of drift is based on the approximation of the option price obtained by either fast mean-reversion or small noise expansions. Fast mean-reversion expansion is the approximation to the option price given by the Black-Scholes price with long-run average volatility in the leading term. Small noise expansion is another approximation to the option price given by the Black-Scholes price with constant volatility. Fast mean-reversion expansion works well for mean-reversion rate being large and small noise expansion works well for mean-reversion rate being small. Fouque and Tullie (2002) apply importance sampling using fast mean-reversion and small noise expansion for 
European option in the Scott's stochastic volatility model and Tullie (2002) also to American and barrier options. Importance sampling for pricing barrier options under the Black-Scholes model and LIBOR market model was used by Glasserman and Staum (2001). Joshi and Leung (2007) also apply importance sampling to speed up pricing of barrier options in the Merton jump-diffusion model. Fouque and Han (2004) apply importance sampling to European as well as Asian options in a 2-factor stochastic volatility model. Robertson (2010) considers the Heston model and uses Sample Path Large Deviation Principles to identify an asymptotically optimal change of drift that is deterministic. It is defined as a solution to a 2-dimensional variational problem that reduces to an Euler-Lagrange equation. This is very much in the spirit of Guasoni and Robertson (2008), with the difference that the variational problem is 2-dimensional and the corresponding Euler-Lagrange equation is more complicated.

\section{$3 \quad$ Heston with Importance Sampling}

The dynamics of the Heston model under the risk neutral measure $\mathbb{Q}$ is given by

$$
\begin{aligned}
d S_{t} & =r S_{t} d t+\sqrt{v_{t}} S_{t} d W_{t}^{S} \\
d v_{t} & =\kappa\left(\theta-v_{t}\right) d t+\xi \sqrt{v_{t}} d W_{t}^{v}
\end{aligned}
$$

where $\left\langle d W_{t}^{S}, d W_{t}^{v}\right\rangle=\rho d t . S_{t}$ is the stock price, $r$ is the risk-free interest rate, $v_{t}$ is the variance, $\kappa$ is the mean-reversion rate, $\theta$ is the long-term variance, $\xi$ is the volatility of volatility, and $\rho$ is the correlation between stock returns and changes in the variance.

In matrix notation, the dynamics in (1) and (2) can be represented as

$$
d X_{t}=b\left(X_{t}\right) d t+a\left(X_{t}\right) d \eta_{t}
$$

where $\eta_{t}$ is a 2 -dimensional $\mathbb{Q}$-Brownian motion and

$$
\begin{aligned}
& d X_{t}=\left(\begin{array}{c}
S_{t} \\
v_{t}
\end{array}\right) \\
& b(x)=\left(\begin{array}{c}
r s \\
\kappa(\theta-v)
\end{array}\right)
\end{aligned}
$$




$$
\begin{aligned}
a(x) & =\left(\begin{array}{cc}
\sqrt{v} s & 0 \\
\xi \sqrt{v} \rho & \xi \sqrt{v\left(1-\rho^{2}\right)}
\end{array}\right) \\
\eta_{t} & =\left(\begin{array}{c}
W_{t}^{S} \\
W_{t}^{v}
\end{array}\right)
\end{aligned}
$$

In this setup, the price $P\left(t, X_{t}\right)$ of a European option at time $t$ is given by

$$
P(t, x)=\mathbb{E}\left[e^{-r(T-t)} \max \left(S_{T}-K\right) \mid X_{t}=x\right]
$$

Here $T$ is the option maturity, $r$ is the risk-free interest rate, $K$ is the strike price, and $S_{T}$ is the price of the underlying asset at $T$.

\subsection{Changing Measure}

Following Fouque and Tullie (2002), we derive the optimal change of measure for the Heston model.

First, we introduce the martingale

$$
H_{t}=\exp \left(\int_{0}^{T} h^{\top}\left(s, X_{s}\right) d \eta_{s}+\frac{1}{2} \int_{0}^{T}\left\|h\left(s, X_{s}\right)\right\|^{2} d s\right)
$$

where $h^{\top}$ denotes the transpose of $h$. Next, we define a new probability measure denoted by $\tilde{\mathbb{Q}}$ which is equivalent to $\mathbb{Q}$ by its Radon-Nikodym derivative

$$
\frac{d \tilde{\mathbb{Q}}}{d \mathbb{Q}}=\left(H_{T}\right)^{-1}
$$

By the Girsanov theorem, the process

$$
\tilde{\eta}_{t}=\eta_{t}+\int_{0}^{t} h\left(s, X_{s}\right) d \eta_{s}
$$

is a 2-dimensional $\tilde{\mathbb{Q}}$-Brownian motion. Using $\tilde{\eta}_{t},(3)$ and (4) can be written as

$$
\begin{aligned}
d X_{t} & =\left(b\left(X_{t}\right)-a\left(X_{t}\right) h\left(t, X_{t}\right)\right) d t+a\left(X_{t}\right) d \tilde{\eta}_{t} \\
H_{t} & =\exp \left(\int_{0}^{T} h^{\top}\left(s, X_{s}\right) d \tilde{\eta}_{t}-\frac{1}{2} \int_{0}^{T}\left\|h\left(s, X_{s}\right)\right\|^{2} d s\right)
\end{aligned}
$$


With respect to the new measure, the price of a European option becomes

$$
P(t, x)=\tilde{\mathbb{E}}\left[e^{-r(T-t)} \max \left(S_{T}-K\right) H_{T} \mid X_{t}=x\right]
$$

Next, we apply Ito's lemma to $P\left(t, X_{t}\right) H_{t}$ and use Kolmogorov backward equation for $P\left(t, X_{t}\right)$

$$
\begin{aligned}
d\left(P\left(t, X_{t}\right) H_{t}\right) & =P\left(t, X_{t}\right) d H_{t}+H_{t} d P\left(t, X_{t}\right)+H_{t} h^{\top} a \nabla P d t \\
& =P H_{t} h^{\top} d \tilde{\eta}_{t}+H_{t}\left(\frac{\partial P}{\partial t} d t+\nabla P^{\top} d X+\frac{1}{2} \operatorname{tr}\left(\nabla^{2} P \cdot a a^{\top}\right) d t\right)+ \\
& +H_{t} h^{\top} a \nabla P d t \\
& =P H_{t} h^{\top} d \tilde{\eta}_{t}+H_{t} \frac{\partial P}{\partial t} d t+H_{t} \nabla P^{\top}\left[(b-a h) d t+a d \tilde{\eta}_{t}\right]+ \\
& +\frac{1}{2} H_{t} \operatorname{tr}\left(\nabla^{2} P \cdot a a^{\top}\right) d t+H_{t} h^{\top} a \nabla P d t \\
& =P H_{t} h^{\top} d \tilde{\eta}_{t}+H_{t} \frac{\partial P}{\partial t} d t+H_{t} \nabla P^{\top} b d t+H_{t} \nabla P^{\top} a d \tilde{\eta}_{t}+ \\
& +\frac{1}{2} H_{t} \operatorname{tr}\left(\nabla^{2} P \cdot a a^{\top}\right) d t \\
& =P H_{t} h^{\top} d \tilde{\eta}_{t}+H_{t} \nabla P^{\top} a d \tilde{\eta}_{t} \\
& =H_{t}\left(\nabla P^{\top} a+P h^{\top}\right) d \tilde{\eta}_{t}
\end{aligned}
$$

where $\nabla P$ is the gradient of $P$ with respect to the state variable $x$. Integrating $d\left(P\left(t, X_{t}\right) H_{t}\right)$ from 0 to $T$ yields

$$
P\left(T, X_{T}\right) H_{T}=P\left(0, X_{0}\right) H_{0}+\int_{0}^{T} H_{t}\left(P\left(t, X_{t}\right) h\left(t, X_{t}\right)^{\top}+\nabla P\left(t, X_{t}\right)^{\top} a\left(t, X_{t}\right)\right) d \tilde{\eta}_{t}
$$

which reduces to

$$
\phi\left(X_{T}\right) H_{T}=P(0, x)+\int_{0}^{T} H_{t}\left(P\left(t, X_{t}\right) h\left(t, X_{t}\right)^{\top}+\nabla P\left(t, X_{t}\right)^{\top} a\left(t, X_{t}\right)\right) d \tilde{\eta}_{t}
$$

where $\phi\left(X_{T}\right)$ is the option payoff at maturity. Therefore, the variances of Monte Carlo estimators under $\tilde{\mathbb{Q}}$ and $\mathbb{Q}$ respectively are given by

$$
\begin{aligned}
\operatorname{Var}_{\tilde{\mathbb{Q}}}\left(\phi\left(X_{T}\right) H_{T}\right) & =\tilde{\mathbb{E}}\left[\int_{0}^{T} H_{t}^{2}\left\|P\left(t, X_{t}\right) h\left(t, X_{t}\right)^{\top}+\nabla P\left(t, X_{t}\right)^{\top} a\left(t, X_{t}\right)\right\|^{2} d t\right] \\
\operatorname{Var}_{\mathbb{Q}}\left(\phi\left(X_{T}\right)\right) & =\mathbb{E}\left[\int_{0}^{T}\left\|\nabla P\left(t, X_{t}\right)^{\top} a\left(t, X_{t}\right)\right\|^{2} d t\right]
\end{aligned}
$$


and the optimal choice of $h$ for which the variance of $\phi\left(X_{T}\right) H_{T}$ under $\tilde{\mathbb{Q}}$ is minimized is

$$
h\left(t, X_{t}\right)=-\frac{1}{P\left(t, X_{t}\right)} a\left(t, X_{t}\right)^{\top} \nabla P\left(t, X_{t}\right)
$$

The difficulty with (6) is that $P\left(t, X_{t}\right)$ and $\nabla P\left(t, X_{t}\right)$ are not known. Indeed, the whole purpose of the simulation is to find $P\left(t, X_{t}\right)$. Hence, the Black-Scholes equivalents of $P\left(t, X_{t}\right)$ and $\nabla P\left(t, X_{t}\right)$ will be used instead. In what follows, we study the conditions under which the Heston model can be approximated by the Black-Scholes model.

Following Fouque, Papanicolaou and Sircar (2011), small noise expansion is obtained by setting $\kappa=\delta$ and $\xi=m \sqrt{2 \delta}$. The dynamics of the Heston model becomes

$$
\begin{aligned}
d S_{t} & =r S_{t} d t+\sqrt{v_{t}} S_{t} d W_{t}^{S} \\
d v_{t} & =\delta\left(\theta-v_{t}\right) d t+m \sqrt{2 \delta v_{t}} d W_{t}^{v}
\end{aligned}
$$

where $\left\langle d W_{t}^{S}, d W_{t}^{v}\right\rangle=\rho d t, \delta$ is a small positive parameter and the Feller condition, $m^{2}<\theta$, ensures that $v_{t}$ is always positive.

This leads to the following fundamental partial differential equation for pricing derivatives

$$
\begin{aligned}
0 & =\frac{\partial P}{\partial t}+\frac{1}{2} v S^{2} \frac{\partial^{2} P}{\partial S^{2}}+\rho m \sqrt{2 \delta} v S \frac{\partial^{2} P}{\partial S \partial v}+m^{2} \delta v \frac{\partial^{2} P}{\partial v^{2}} \\
& +r\left(S \frac{\partial P}{\partial S}-P\right)+[\delta(\theta-v)-\lambda v] \frac{\partial P}{\partial v}
\end{aligned}
$$

which can be written as

$$
\left(\delta \mathcal{M}_{0}+\sqrt{\delta} \mathcal{M}_{1}+\mathcal{M}_{2}\right) P=0
$$

with differential operators

$$
\begin{aligned}
& \mathcal{M}_{0}=m^{2} v \frac{\partial^{2}}{\partial v^{2}}+(\theta-v) \frac{\partial}{\partial v} \\
& \mathcal{M}_{1}=\rho m \sqrt{2} v S \frac{\partial^{2}}{\partial S \partial v} \\
& \mathcal{M}_{2}=\frac{\partial}{\partial t}+\frac{1}{2} v S^{2} \frac{\partial^{2}}{\partial S^{2}}+r\left(S \frac{\partial}{\partial S}-.\right)-\lambda v \frac{\partial}{\partial v}
\end{aligned}
$$

When the market price of volatility risk $\lambda$ is $0, \mathcal{M}_{2}$ is the Black-Scholes operator with volatility $\sqrt{v}$. If $\delta=0,(7)$ becomes

$$
\mathcal{M}_{2} P=0
$$

Therefore, the approximation $P_{S N E}$ of $P\left(t, X_{t}\right)$ is given by the Black-Scholes formula with volatility 
$\sqrt{v}$. When volatility is slowly varying, that is when $\kappa \rightarrow 0$, volatility is stuck at its initial level. In that case, the approximation $P_{S N E}$ of $P\left(t, X_{t}\right)$ is given by the Black-Scholes formula with volatility $\sqrt{v_{0}}$.

As an alternative expansion, the fast mean-reversion is obtained by setting $\kappa=\frac{1}{\varepsilon}$ and $\xi=\frac{m \sqrt{2}}{\sqrt{\varepsilon}}$. The dynamics of the Heston model becomes

$$
\begin{aligned}
d S_{t} & =r S_{t} d t+\sqrt{v_{t}} S_{t} d W_{t}^{S} \\
d v_{t} & =\frac{1}{\varepsilon}\left(\theta-v_{t}\right) d t+\frac{m}{\sqrt{\varepsilon}} \sqrt{2 v_{t}} d W_{t}^{v}
\end{aligned}
$$

where $\left\langle d W_{t}^{S}, d W_{t}^{v}\right\rangle=\rho d t, \varepsilon$ is a small positive parameter and the Feller condition, $m^{2}<\theta$, ensures that $v_{t}$ is always positive.

Similarly, the fundamental partial differential equation for pricing derivatives is

$$
\begin{aligned}
0 & =\frac{\partial P}{\partial t}+\frac{1}{2} v S^{2} \frac{\partial^{2} P}{\partial S^{2}}+\rho \frac{m \sqrt{2}}{\sqrt{\varepsilon}} v S \frac{\partial^{2} P}{\partial S \partial v}+\frac{m^{2}}{\varepsilon} v \frac{\partial^{2} P}{\partial v^{2}} \\
& +r\left(S \frac{\partial P}{\partial S}-P\right)+\left[\frac{1}{\varepsilon}(\theta-v)-\lambda v\right] \frac{\partial P}{\partial v}
\end{aligned}
$$

which can be written as

$$
\left(\frac{1}{\varepsilon} \mathcal{L}_{0}+\frac{1}{\sqrt{\varepsilon}} \mathcal{L}_{1}+\mathcal{L}_{2}\right) P=0
$$

with differential operators

$$
\begin{aligned}
\mathcal{L}_{0} & =m^{2} v \frac{\partial^{2}}{\partial v^{2}}+(\theta-v) \frac{\partial}{\partial v} \\
\mathcal{L}_{1} & =\rho m \sqrt{2} v S \frac{\partial^{2}}{\partial S \partial v} \\
\mathcal{L}_{2} & =\frac{\partial}{\partial t}+\frac{1}{2} v S^{2} \frac{\partial^{2}}{\partial S^{2}}+r\left(S \frac{\partial}{\partial S}-.\right)-\lambda v \frac{\partial}{\partial v}
\end{aligned}
$$

Expanding $P$ in powers of $\sqrt{\varepsilon}$, we get

$$
P=P_{0}+\sqrt{\varepsilon} P_{1}+\cdots
$$


Substituting this into (8) yields

$$
\begin{aligned}
\frac{1}{\varepsilon} \mathcal{L}_{0} P_{0} & +\frac{1}{\sqrt{\varepsilon}}\left(\mathcal{L}_{0} P_{1}+\mathcal{L}_{1} P_{0}\right) \\
& +\left(\mathcal{L}_{2} P_{0}+\mathcal{L}_{1} P_{1}\right) \\
& +\sqrt{\varepsilon} \mathcal{L}_{2} P_{1} \\
& +\cdots \\
& =0
\end{aligned}
$$

We eliminate terms of order $\frac{1}{\varepsilon}, \frac{1}{\sqrt{\varepsilon}}, \sqrt{\varepsilon}$ by equating them to 0 . This leads to

$$
\left(\mathcal{L}_{2} P_{0}+\mathcal{L}_{1} P_{1}\right)=0
$$

Since the operator $\mathcal{L}_{1}$ takes derivatives with respect to $v$ and $P_{1}$ must be constant with respect to $v$, we must have $\mathcal{L}_{1} P_{1}=0$. Finally, we have

$$
\mathcal{L}_{2} P_{0}=0
$$

When the market price of volatility risk $\lambda$ is $0, \mathcal{L}_{2}$ is the Black-Scholes operator with constant volatility. As shown in Fouque, Papanicolaou and Sircar (2011), $P_{0}=P_{B S(\bar{\sigma})}$ where $\bar{\sigma}$ is the constant volatility $\bar{\sigma}=\sqrt{\theta}$. Therefore, the approximation $P_{F M R}$ of $P\left(t, X_{t}\right)$ is given by the BlackScholes formula with volatility $\sqrt{\theta}$.

From (6), under the small noise expansion, $h$ is given by

$$
h=-\frac{1}{P_{S N E}}\left(\begin{array}{c}
s \sqrt{v} \frac{\partial P_{S N E}}{\partial s} \\
0
\end{array}\right)
$$

where $P_{S N E}$ is the option price under the classic geometric Brownian motion dynamics with volatility $\sqrt{v_{0}}$. Similarly, under the fast mean-reversion expansion, $h$ is given by

$$
h=-\frac{1}{P_{F M R}}\left(\begin{array}{c}
s \sqrt{v} \frac{\partial P_{F M R}}{\partial s} \\
0
\end{array}\right)
$$

where $P_{F M R}$ is the option price under the classic geometric Brownian motion dynamics with volatility $\sqrt{\theta}$. 
To simulate the Heston model using importance sampling we use the following discretization

$$
\begin{aligned}
S_{i+1}= & S_{i} \exp \left(\left(r-\frac{v_{i}}{2}-\sqrt{v_{i}} h\left(i, S_{i}, v_{i}\right)\right) \Delta t+\sqrt{v_{i} \Delta t} W_{i+1}^{S}\right) \\
v_{i+1}= & v_{i}+\left(\kappa\left(\theta-v_{i}\right)-\sqrt{v_{i}} \xi \rho h\left(i, S_{i}, v_{i}\right)\right) \Delta t \\
& +\xi \sqrt{v_{i} \Delta t}\left(\rho W_{i+1}^{S}+\sqrt{1-\rho^{2}} W_{i+1}^{v}\right)
\end{aligned}
$$

where $\Delta t=t_{i+1}-t_{i}, W_{i+1}^{S}$ and $W_{i+1}^{v}$ are standard normal random variables. Then from (5) the option payoff is multiplied by the Radon-Nikodym derivative of the risk neutral probability measure with respect to the importance sampling measure

$$
H_{T}=\exp \left(\sum_{i=1}^{M} h\left(i, S_{i}, v_{i}\right) \sqrt{\Delta t} W_{i+1}^{S}-\frac{1}{2} \sum_{i=1}^{M} h\left(i, S_{i}, v_{i}\right)^{2} \Delta t\right)
$$

where $M$ is the number of time steps.

\subsection{Numerical Examples}

In this section, we present the numerical results for a European call, where a semi-analytical solution exists, and for a down-and-out put, where an analytical solution is not available. Here, we set $\rho=-0.4, \xi=0.4, v_{0}=0.04, \theta=0.09, S_{0}=100, r=5 \%$, and $T=1$ year. The barrier for the down-and-out put is set at 50 . Initial variance of 0.04 corresponds to volatility of 0.2 , whereas long-run variance of 0.09 corresponds to volatility of 0.3 . We tested four rates of mean reversion, $\kappa: 0.5,2,5,10$ and five strikes, $K: 60,80,100,120,140$. As benchmarks, we compared option prices simulated under the importance sampling (Small Noise Expansion, SNE, and Fast MeanReversion, FMR) against the basic Monte Carlo (MC), control variate (CV), and semi-analytical solution $(\mathrm{H})$. All simulations are performed using the same sequence of pseudo-random numbers. We simulate 10,000 sample paths. For the European call we use a time-increment of 0.001, whereas for the down-and-out put we use a time-increment of $1 / 252$, which corresponds to one business day. As the control variate, we use the classic geometric Brownian motion dynamics with a volatility of $30 \%$ which is the long-run mean of volatility used for the Heston model.

Table 1 presents the price, variance, and relative error of the European call. The relative error is measured against the semi-analytical solution. Table 2 presents the price and variance for the down-and-out put option, where analytical solution is not available. For the European call, the importance sampling strongly outperforms the basic MC in terms of variance reduction and price accuracy. Variance reduction for SNE is up to 105 times compared to MC. For FMR, this is up to 880 times, whereas for $\mathrm{CV}$ this is up to 35 times. The amount of variance reduction 
depends on mean-reversion rate and strike. Greater variance reduction is achieved with higher mean-reversion rate and the more the option is in-the-money. For the European call, CV has comparable performance as the importance sampling; it is more efficient than SNE but inferior to FMR. However, for the barrier option, the superiority of the importance sampling continues, but CV starts to deteriorate especially for in-the-money put option. Note that as we do not have the analytical solution for the barrier option price under the Heston model, we are not able to conclude on accuracy. Between the two importance sampling schemes, FMR is more efficient than SNE even for cases when mean-reversion speed is slow. Fouque and Tullie (2002) report a similar finding for the Scott's model.

So far, we show that importance sampling outperformed both $\mathrm{MC}$ and $\mathrm{CV}$ in terms of variance reduction especially for barrier option. However, SNE and FMR are computationally expensive, as the change of the drift depends on option price and delta in the Black-Scholes model at every time step. For example, if each sample path is simulated using 1,000 time steps and there are 10,000 sample paths, one has to calculate 10,000,000 Black-Scholes prices and deltas. There is no such calculation in MC, whereas CV requires the simulation of 10,000 sample paths to obtain the Monte Carlo estimate of the option price used as a control variate. Table 3 reports price, variance of price, and computational time for an at-the-money European call option, when the mean-reversion is 0.5. Variance reduction ratio and speed are measured against the basic Monte Carlo. Effective performance is defined as variance reduction ratio over speed. Speed itself is defined as the ratio of computational time of the importance sampling to computational time of the basic Monte Carlo.

We note from Table 3 that FMR reduces the variance almost 10 times compared to MC, but it is 120 times slower. SNE reduces the variance 6 times compared to MC, but it is also 120 times slower. Therefore, the effective performance of both FMR and SNE is far worse than that of MC. On the other hand, the effective performance of $\mathrm{CV}$ is almost 2 times better than MC.

To improve the effective performance of FMR and SNE, we propose the use of a lookup table. As mentioned before, when calculating the drift in the simulation we repeatedly re-calculate the Black-Scholes price and delta at every time step for different pairs of stock price and time to maturity. Therefore, it is possible to construct a 2-dimensional lookup table using the range of possible values of the stock price and the time to maturity according to the time step used in the simulation. This way the number of times Black-Scholes prices and deltas are calculated is significantly reduced. 
莡

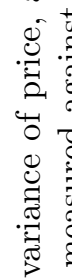

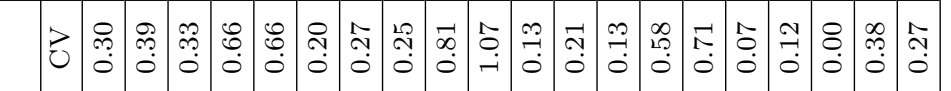

8

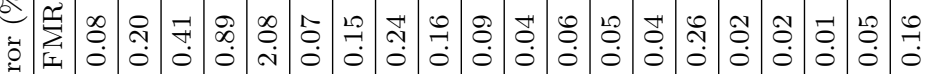

总坛

حi.

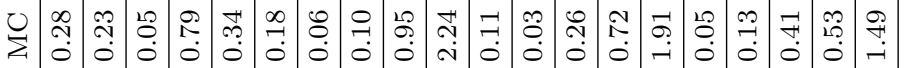

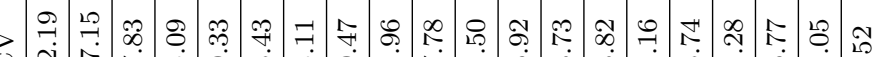

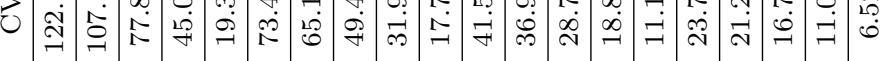

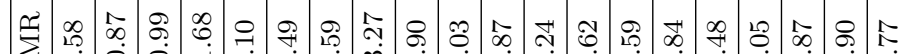

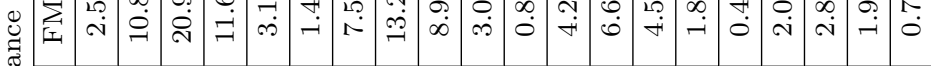

䒕

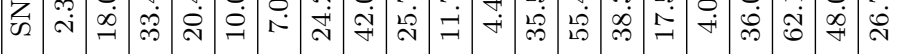

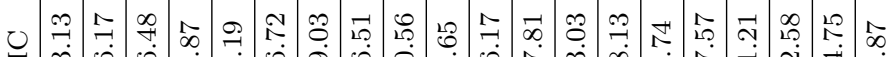

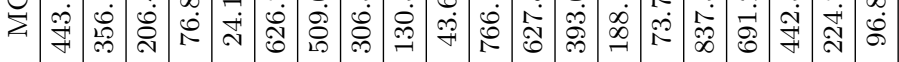

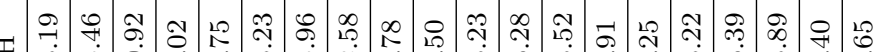

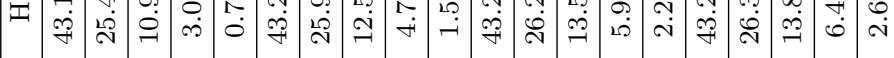

$>$ ๖

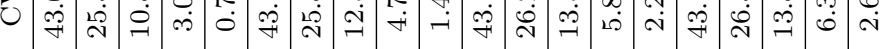

•

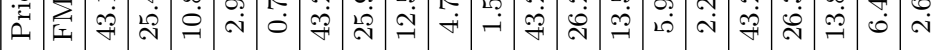

四 8 ○

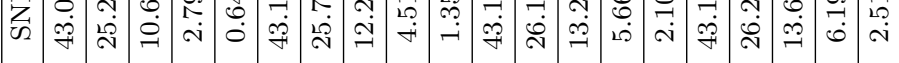

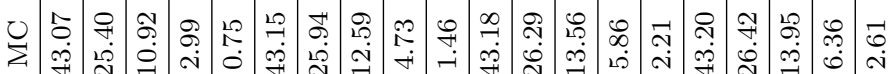

k

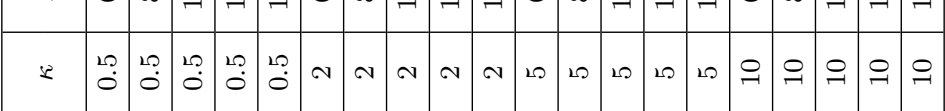

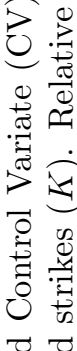

䔡

종

至紫

음.

㱐

完

尝

तै

宫㝵

芴:

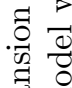

을

甾

啹

西

影

0

$\leq \mathrm{i}$

율 Ð

章量高

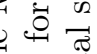

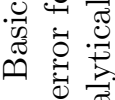




\begin{tabular}{|c|c|c|c|c|c|c|c|c|c|}
\hline \multirow{2}{*}{$\kappa$} & \multirow{2}{*}{$K$} & \multicolumn{9}{|c|}{ Price } & \multicolumn{4}{c|}{ Variance } \\
\cline { 3 - 10 } & & MC & SNE & FMR & CV & MC & SNE & FMR & CV \\
\hline 0.5 & 60 & 0.06 & 0.02 & 0.03 & 0.06 & 0.31 & 0.03 & 0.11 & 0.30 \\
\hline 0.5 & 80 & 1.04 & 0.81 & 0.95 & 1.02 & 14.67 & 5.95 & 4.29 & 10.47 \\
\hline 0.5 & 100 & 5.15 & 4.87 & 4.99 & 5.06 & 91.72 & 33.69 & 13.09 & 44.49 \\
\hline 0.5 & 120 & 15.89 & 15.56 & 15.69 & 15.75 & 241.87 & 44.03 & 40.04 & 91.40 \\
\hline 0.5 & 140 & 32.26 & 31.98 & 32.02 & 32.09 & 359.81 & 51.44 & 39.98 & 148.68 \\
\hline 2 & 60 & 0.08 & 0.03 & 0.05 & 0.08 & 0.42 & 0.21 & 0.03 & 0.37 \\
\hline 2 & 80 & 1.47 & 1.25 & 1.37 & 1.43 & 19.73 & 15.21 & 1.76 & 9.83 \\
\hline 2 & 100 & 6.70 & 6.42 & 6.55 & 6.58 & 116.03 & 42.20 & 9.97 & 34.99 \\
\hline 2 & 120 & 17.56 & 17.25 & 17.28 & 17.39 & 296.60 & 60.26 & 22.62 & 71.92 \\
\hline 2 & 140 & 32.89 & 32.57 & 32.58 & 32.68 & 472.43 & 61.23 & 32.29 & 119.85 \\
\hline 5 & 60 & 0.08 & 0.05 & 0.07 & 0.08 & 0.43 & 0.36 & 0.01 & 0.34 \\
\hline 5 & 80 & 1.80 & 1.63 & 1.71 & 1.76 & 23.35 & 26.05 & 0.98 & 7.94 \\
\hline 5 & 100 & 7.70 & 7.45 & 7.51 & 7.57 & 131.74 & 70.67 & 5.29 & 26.91 \\
\hline 5 & 120 & 18.76 & 18.45 & 18.47 & 18.57 & 327.30 & 74.11 & 12.60 & 56.02 \\
\hline 5 & 140 & 33.76 & 33.37 & 33.41 & 33.53 & 529.30 & 66.55 & 21.71 & 93.07 \\
\hline 10 & 60 & 0.09 & 0.06 & 0.08 & 0.09 & 0.43 & 0.89 & 0.01 & 0.26 \\
\hline 10 & 80 & 1.95 & 1.80 & 1.87 & 1.90 & 24.96 & 27.15 & 0.58 & 5.07 \\
\hline 10 & 100 & 8.17 & 7.91 & 7.99 & 8.02 & 138.33 & 74.28 & 3.26 & 17.00 \\
\hline 10 & 120 & 19.37 & 19.05 & 19.11 & 19.17 & 339.36 & 84.77 & 8.40 & 35.38 \\
\hline 10 & 140 & 34.32 & 33.96 & 33.96 & 34.07 & 549.13 & 72.73 & 15.74 & 58.47 \\
\hline
\end{tabular}

Table 2: Basic Monte Carlo (MC), Small Noise Expansion (SNE), Fast Mean-Reversion (FMR), and Control Variate (CV) price and variance of price for a down-and-out put under the Heston model with different mean-reversion rates $(\kappa)$ and strikes $(K)$.

To demonstrate the performance of this numerical scheme, we used a 2-dimensional lookup table with 451 stock prices $[50,51, \ldots, 500]$ and 1000 times to maturity $[1,0.999, \ldots, 0.001]$, which corresponds to the 1,000 time steps in the Monte Carlo simulation. Table 3 shows that using the lookup table slightly increased the variance of SNE and FMR. As reported in Table 3, the use of a lookup table speeds up SNE and FMR by 60 times. Note that the computational time reported in Table 3 for SNE and FMR with a lookup table includes time needed to generate the lookup table. It is clear that the lookup table improves the effective performance of SNE and FMR to the point that not only they significantly outperform MC but also CV.

\section{Adding Jumps}

Here, we demonstrate how the importance sampling can still be used when a jump process is added to the dynamics in (1). To this end, we follow the stochastic volatility with jump dynamics in Bates (1996). The dynamics of the Bates model under the risk neutral measure denoted by $\mathbb{Q}$ is 


\begin{tabular}{|c|c|c|c|c|c|c|}
\hline & Price & Variance & $\begin{array}{c}\text { Time } \\
(\mathrm{sec})\end{array}$ & $\begin{array}{c}\text { Variance } \\
\text { reduction } \\
\text { ratio }\end{array}$ & Speed & $\begin{array}{c}\text { Effective } \\
\text { performance }\end{array}$ \\
\hline MC & 10.92 & 206.48 & 0.51 & 1 & 1 & 1 \\
\hline CV & 10.88 & 77.83 & 0.71 & 2.65 & 1.39 & 1.91 \\
\hline SNE & 10.63 & 33.42 & 62.62 & 6.18 & 123.05 & 0.05 \\
\hline FMR & 10.88 & 20.99 & 62.27 & 9.84 & 122.36 & 0.08 \\
\hline SNE with lookup table & 10.58 & 37.96 & 1.18 & 5.44 & 2.32 & 2.34 \\
\hline FMR with lookup table & 10.86 & 21.8 & 1.23 & 9.47 & 2.41 & 3.93 \\
\hline
\end{tabular}

Table 3: Performance measures for basic Monte Carlo (MC), Control Variate (CV), Small Noise Expansion (SNE), and Fast Mean-Reversion (FMR).

given by

$$
\begin{aligned}
d S_{t} & =S_{t}(r-\lambda \bar{k}) d t+S_{t} \sqrt{v_{t}} d W_{t}^{S}+S_{t} d Z_{t} \\
d v_{t} & =\kappa\left(\theta-v_{t}\right) d t+\xi \sqrt{v_{t}} d W_{t}^{v}
\end{aligned}
$$

where $\left\langle d W_{t}^{S} d W_{t}^{v}\right\rangle=\rho d t . S_{t}$ is the stock price, $r$ is the risk-free interest rate, $v_{t}$ is the variance, $\kappa$ is the mean-reversion rate, $\theta$ is the long-term variance, $\xi$ is the volatility of volatility, and $\rho$ is the correlation between stock returns and changes in the variance. $Z_{t}$ is a compound Poisson process with intensity $\lambda$ and log-normal distribution of jump sizes such that if $k$ is its jump size then $\ln (1+k) \sim \mathcal{N}\left(\ln (1+\bar{k})-\frac{1}{2} \delta^{2}, \delta^{2}\right)$.

For the purpose of importance sampling, we will rewrite the model as follows

$$
\begin{aligned}
d S_{t} & =S_{t}(r-\lambda \bar{k}) d t+S_{t}\left(\sqrt{v_{t}}+\frac{d Z_{t}}{d W_{t}^{S}}\right) d W_{t}^{S} \\
d v_{t} & =\kappa\left(\theta-v_{t}\right) d t+\xi \sqrt{v_{t}} d W_{t}^{v}
\end{aligned}
$$

Comparing (9) and (11), it is clear that the dynamics is the same.

In matrix notation the model dynamics is

$$
d X_{t}=b\left(X_{t}\right) d t+a\left(X_{t}\right) d \eta_{t}
$$


where $\eta_{t}$ is a 2-dimensional $\mathbb{Q}$-Brownian motion and

$$
\begin{aligned}
& d X_{t}=\left(\begin{array}{c}
S_{t} \\
v_{t}
\end{array}\right) \\
& b(x)=\left(\begin{array}{c}
s(r-\lambda \bar{k}) \\
\kappa(\theta-v)
\end{array}\right) \\
& a(x)=\left(\begin{array}{cc}
s\left(\sqrt{v}+\frac{d Z_{t}}{d W_{t}^{S}}\right) & 0 \\
\xi \sqrt{v} \rho & \xi \sqrt{v\left(1-\rho^{2}\right)}
\end{array}\right) \\
& \eta_{t}=\left(\begin{array}{c}
W_{t}^{S} \\
W_{t}^{v}
\end{array}\right)
\end{aligned}
$$

\subsection{Importance sampling}

Using the analogous derivation to that presented in Section 3, the optimal choice of $h$ for which the variance is minimized has the same form as (6). With the Bates model in (13) and from (6), under the small noise expansion, $h$ is given by

$$
h=-\frac{1}{P_{S N E}}\left(\begin{array}{c}
s\left(\sqrt{v}+\frac{d Z}{d W^{S}}\right) \frac{\partial P_{S N E}}{\partial s} \\
0
\end{array}\right)
$$

where $P_{S N E}$ is the option price under the classic geometric Brownian motion dynamics with volatility $\sqrt{v_{0}}$. Similarly, under the fast mean-reversion expansion, $h$ is given by

$$
h=-\frac{1}{P_{F M R}}\left(\begin{array}{c}
s\left(\sqrt{v}+\frac{d Z}{d W^{S}}\right) \frac{\partial P_{F M R}}{\partial s} \\
0
\end{array}\right)
$$

where $P_{F M R}$ is the option price under the classic geometric Brownian motion dynamics with volatility $\sqrt{\theta}$.

To simulate the Bates model using importance sampling we use the following discretization

$$
\begin{aligned}
S_{i+1}= & S_{i} \exp \left(\mu \Delta t+\sqrt{v_{i} \Delta t} W_{i+1}^{S}+\sum_{j=1}^{N_{i}} k_{j}\right) \\
\mu= & \left(r-\lambda \bar{k}-\frac{v_{i}}{2}-\sqrt{v_{i}}\left(h\left(i, S_{i}, v_{i}\right)+\frac{\sum_{j=1}^{N_{i}} k_{j}}{\sqrt{\Delta t} W_{i+1}^{S}}\right)\right) \\
v_{i+1}= & v_{i}+\left(\kappa\left(\theta-v_{i}\right)-\sqrt{v_{i}} \xi \rho h\left(i, S_{i}, v_{i}\right)\right) \Delta t \\
& +\xi \sqrt{v_{i} \Delta t}\left(\rho W_{i+1}^{S}+\sqrt{1-\rho^{2}} W_{i+1}^{v}\right)
\end{aligned}
$$


where $\Delta t=t_{i+1}-t_{i}, W_{i+1}^{S}$ and $W_{i+1}^{v}$ are standard normal random variables, $N_{i}$ is a Poisson random variable with mean $\lambda \Delta t$, and $\ln (1+k) \sim \mathcal{N}\left(\left(\ln (1+\bar{k})-\frac{1}{2} \delta^{2}\right), \delta^{2}\right)$. If $W_{i+1}^{S}$ happens to be 0 , we set $\frac{\sum_{j=1}^{N_{i}} k_{j}}{\sqrt{\Delta t} W_{i+1}^{S}}$ to 0 , which means that we apply importance sampling only to the stochastic volatility component. Then from (5) the option payoff is multiplied by the Radon-Nikodym derivative of the risk neutral probability measure with respect to the importance sampling measure. Here $H_{T}$ has the same form as (6), but $h$ follows that in (14) for SNE scheme and (15) for FMR scheme.

\subsection{Numerical Examples}

As in the previous section, the price is obtained using five methods: basic Monte Carlo (MC), Small Noise Expansion (SNE), Fast Mean-Reversion (FMR), Control Variate (CV), and semianalytical solution (B). We assume that the jump intensity is 1 jump per year, standard deviation of the jumps is $2 \%$, and the mean jump size is $-5 \%$. All other parameters are the same as in the Heston model in Section 3.2. As the control variate, we use the classic geometric Brownian motion dynamics with a volatility of $30 \%$.

Table 4 presents the price, variance, and relative error for the European call. Relative error is measured against the semi-analytical solution. Importance sampling outperforms CV in terms of both variance reduction and relative error. Variance reduction for SNE is up to 27 times compared to MC. For FMR, this is up to 60 times, whereas for CV this is up to 17 times. As before, the amount of variance reduction depends on mean-reversion rate and moneyness. As the meanreversion rate increases and as the option becomes deeper in-the-money, so does the amount of variance reduction. FMR appears to be not only more efficient but also more accurate than than SNE.

Table 5 reports the price and variance for the down-and-out put option. The price is obtained using four methods: basic Monte Carlo (MC), Small Noise Expansion (SNE), Fast Mean-Reversion (FMR), and Control Variate (CV). There is no analytical solution for a barrier option price under the Bates model. The barrier is set at 50 and we use time increment of $1 / 252$, which corresponds to one business day. All the other parameters are the same as those for the European call. Since we do not have the analytic solution, we are not able to conclude on price accuracy. However, it is clear from Table 5 that importance sampling outperforms CV in terms of variance reduction. Variance reduction for SNE is up to 11 times compared to MC. For FMR, this is up to 42 times, whereas for $\mathrm{CV}$ this is up to 6 times. As the mean-reversion rate increases and as the option becomes deeper in-the-money, so does the amount of variance reduction. For the barrier option, we noted that the performance of SNE starts to be less competitive especially for fast mean-reversion and deep in-the-money cases, whereas FMR continues to dominate CV. 


\section{Greeks}

In this section, we show how the Greeks, or derivative hedge ratios, can be calculated by exploiting the importance sampling. Broadie and Kaya (2004) compute Greeks for affine models, including the Heston and the Bates models, using the Likelihood Ratio Method conditioned on the state variables. Glasserman and Liu (2010) also use the Likelihood Ratio Method to calculate the Greeks, but they calculate the likelihood ratio by inverting the Laplace transform of the distribution function using the Abate-Whitt (1992) algorithm. Here, we also use the Likelihood Ratio Method, but one that is based on the characteristic function obtained via Fourier inversion. For comparison, we calculated the Greeks according to the method of Benhamou (2002) which is based on the Malliavin calculus and implemented for the Heston model.

We begin with an option price under $\mathbb{Q}$ defined as

$$
P(t, x)=\int_{0}^{\infty} e^{-r(T-t)} \phi\left(S_{T}\right) f(x) d x
$$

where $\phi\left(S_{T}\right)$ is the payoff function and $f(x)$ is the risk-neutral probability density function.

Next, consider delta, $\Delta$, the first derivative of the option price with respect to $S_{0}$

$$
\begin{aligned}
\Delta & =\frac{\partial}{\partial S_{0}} \int_{0}^{\infty} e^{-r(T-t)} \phi\left(S_{T}\right) f(x) d x \\
& =\int_{0}^{\infty} e^{-r(T-t)} \phi\left(S_{T}\right) \frac{\frac{\partial}{\partial S_{0}} f(x)}{f(x)} f(x) d x
\end{aligned}
$$

where $\frac{\frac{\partial}{\partial S_{0}} f(x)}{f(x)}$ is the likelihood ratio. The probability density function is obtained from the characteristic function using the following inversion formula

$$
\operatorname{Pr}\left(S_{T}>x\right)=\frac{1}{2}+\frac{1}{\pi} \int_{0}^{\infty} \operatorname{Re}\left[\frac{\exp (-i \omega \ln (x)) \psi_{T}(\omega)}{i \omega}\right] d \omega
$$

where $\psi$ is the characteristic function. We note that the characteristic functions for both the Heston and the Bates models are available in semi-closed form. Since the total probability mass is 1 ,

$$
F(x)=\operatorname{Pr}\left(S_{T} \leq x\right)=\frac{1}{2}-\frac{1}{\pi} \int_{0}^{\infty} \operatorname{Re}\left[\frac{\exp (-i \omega \ln (x)) \psi_{T}(\omega)}{i \omega}\right] d \omega
$$

Then the integral in (17) can be calculated using the Gauss-Laguerre quadrature. Finally, to get 


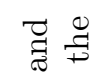

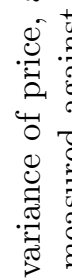

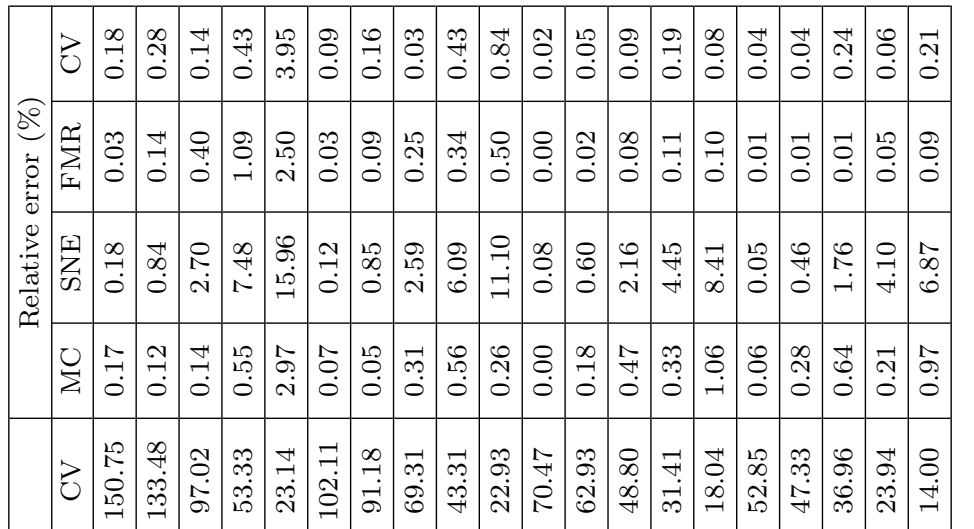

i.

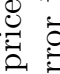

远

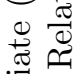

定

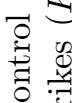

o

荬

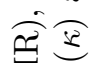

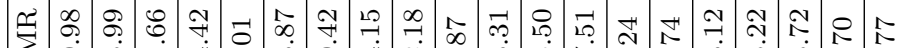

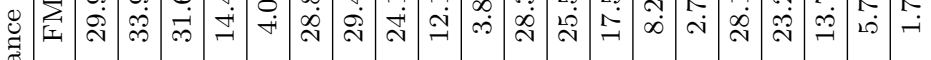

$\stackrel{\varpi}{\sim}$ 四

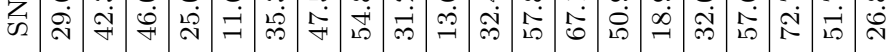

అ

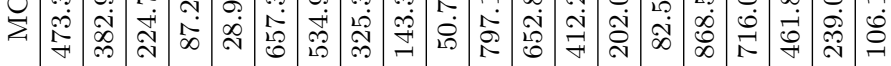

๓

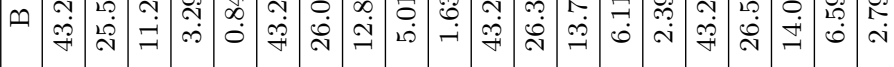

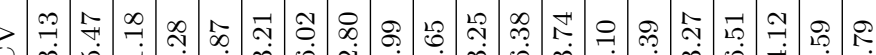

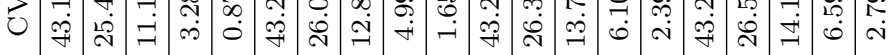

:

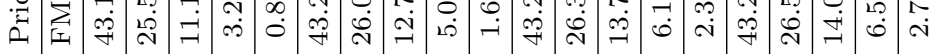

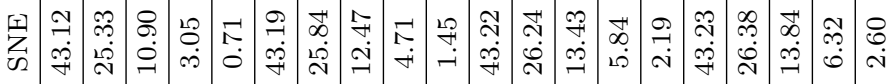

৩

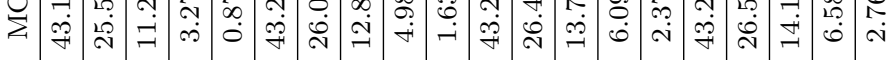

니 $\&$ ○

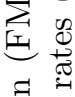

苾

$\approx$

密

㱐

范蓠

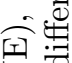

$\sum_{0 .}$

畨

을

甾

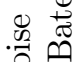

完

鄫

㱐

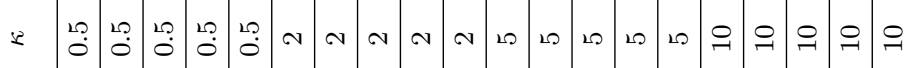

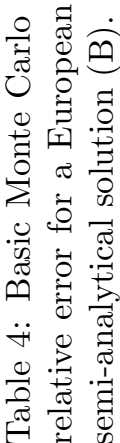




\begin{tabular}{|c|c|c|c|c|c|c|c|c|c|}
\hline \multirow{2}{*}{$\kappa$} & \multirow{2}{*}{$K$} & \multicolumn{6}{|c|}{ Price } & \multicolumn{4}{c|}{ Variance } \\
\cline { 3 - 10 } & & MC & SNE & FMR & CV & MC & SNE & FMR & CV \\
\hline 0.5 & 60 & 0.05 & 0.02 & 0.04 & 0.05 & 0.28 & 0.02 & 0.08 & 0.26 \\
\hline 0.5 & 80 & 1.07 & 0.85 & 0.97 & 1.04 & 14.71 & 7.82 & 4.38 & 10.69 \\
\hline 0.5 & 100 & 5.32 & 5.04 & 5.18 & 5.23 & 93.33 & 37.08 & 17.63 & 48.79 \\
\hline 0.5 & 120 & 16.00 & 15.70 & 15.79 & 15.87 & 248.11 & 64.98 & 48.87 & 107.23 \\
\hline 0.5 & 140 & 32.16 & 31.89 & 31.95 & 32.00 & 375.69 & 83.41 & 70.81 & 171.73 \\
\hline 2 & 60 & 0.07 & 0.03 & 0.05 & 0.07 & 0.37 & 0.12 & 0.04 & 0.33 \\
\hline 2 & 80 & 1.49 & 1.29 & 1.41 & 1.46 & 19.75 & 17.09 & 2.41 & 11.08 \\
\hline 2 & 100 & 6.78 & 6.52 & 6.66 & 6.66 & 117.17 & 57.88 & 14.77 & 42.54 \\
\hline 2 & 120 & 17.60 & 17.26 & 17.35 & 17.43 & 300.00 & 74.41 & 37.27 & 90.45 \\
\hline 2 & 140 & 32.80 & 32.50 & 32.50 & 32.59 & 481.93 & 97.61 & 59.16 & 148.48 \\
\hline 5 & 60 & 0.08 & 0.04 & 0.07 & 0.08 & 0.44 & 0.24 & 0.01 & 0.37 \\
\hline 5 & 80 & 1.83 & 1.63 & 1.74 & 1.79 & 23.62 & 25.35 & 1.61 & 9.77 \\
\hline 5 & 100 & 7.73 & 7.46 & 7.57 & 7.60 & 132.99 & 71.40 & 9.90 & 35.28 \\
\hline 5 & 120 & 18.75 & 18.39 & 18.48 & 18.56 & 330.08 & 91.17 & 26.72 & 74.06 \\
\hline 5 & 140 & 33.64 & 33.26 & 33.29 & 33.41 & 536.72 & 96.43 & 46.91 & 120.80 \\
\hline 10 & 60 & 0.09 & 0.06 & 0.08 & 0.09 & 0.43 & 0.56 & 0.01 & 0.34 \\
\hline 10 & 80 & 1.98 & 1.82 & 1.90 & 1.94 & 25.27 & 33.47 & 1.19 & 8.31 \\
\hline 10 & 100 & 8.20 & 7.89 & 8.03 & 8.05 & 139.63 & 73.70 & 8.04 & 28.64 \\
\hline 10 & 120 & 19.36 & 19.00 & 19.08 & 19.16 & 342.03 & 97.97 & 22.62 & 58.79 \\
\hline 10 & 140 & 34.22 & 33.80 & 33.83 & 33.97 & 555.95 & 98.68 & 40.70 & 94.48 \\
\hline
\end{tabular}

Table 5: Basic Monte Carlo (MC), Small Noise Expansion (SNE), Fast Mean-Reversion (FMR), and Control Variate (CV) price and variance of price for a down-and-out put under the Bates model with different mean-reversion rates $(\kappa)$ and strikes $(K)$.

the probability density function we use the following finite difference approximation

$$
f(x) \approx \frac{\operatorname{Pr}\left(S_{T} \leq x+\Delta x\right)-\operatorname{Pr}\left(S_{T} \leq x\right)}{\Delta x}
$$

To apply importance sampling, note that option price under $\tilde{\mathbb{Q}}$ is

$$
P(t, x)=\int_{0}^{\infty} e^{-r(T-t)} \phi\left(S_{T}\right) \frac{f(x)}{g(x)} g(x) d x
$$

Delta of an option under the importance sampling measure will be obtained by multiplying the integrand of delta under the risk-neutral measure in (16) by $\frac{g(x)}{g(x)}$.

$$
\begin{aligned}
\Delta & =\int_{0}^{\infty} e^{-r(T-t)} \phi\left(S_{T}\right) \frac{\frac{\partial f(x)}{\partial S_{0}}}{f(x)} f(x) \frac{g(x)}{g(x)} d x \\
& =\int_{0}^{\infty} e^{-r(T-t)} \phi\left(S_{T}\right) \frac{\frac{\partial f(x)}{\partial S_{0}}}{f(x)} \frac{f(x)}{g(x)} g(x) d x
\end{aligned}
$$

where $\frac{f(x)}{g(x)}$ is the Radon-Nikodym derivative. Similarly, delta of the down-and-out put option can 
be calculated as

$$
\Delta=\int_{0}^{\infty} e^{-r(T-t)} \phi\left(S_{T}\right) \mathbf{1}\left[\min _{\substack{1 \leq i \leq m \\ S_{i}>B}} B\right] \frac{\frac{\partial f(x)}{\partial S_{0}}}{f(x)} \frac{f(x)}{g(x)} g(x) d x
$$

The same procedure as above can be followed to compute the other Greeks.

For comparison, we compute the Greeks using the Malliavin calculus, where the Greek is an expectation of the payoff times a Malliavin weight

$$
\text { Greek }=\mathbb{E}\left[\phi\left(S_{T}\right) \text { weight }\right]
$$

The Malliavin weights for delta, $\Delta$, and gamma, $\Gamma$, of European option are given by

$$
\begin{aligned}
\text { weight }_{\Delta} & =\frac{1}{S_{o} T} \int_{0}^{T} \frac{d W_{t}^{S}}{\sqrt{v_{t}}} \\
\text { weight }_{\Gamma} & =-\frac{1}{S_{o}^{2} T} \int_{0}^{T} \frac{d W_{t}^{S}}{\sqrt{v_{t}}}+\frac{1}{\left(S_{o} T\right)^{2}}\left(\left(\int_{0}^{T} \frac{d W_{t}^{S}}{\sqrt{v_{t}}}\right)^{2}-\left(\int_{0}^{T} \frac{d t}{v_{t}}\right)\right)
\end{aligned}
$$

\subsection{Heston Greeks}

In this section, we present delta, $\Delta$, and gamma, $\Gamma$, of the Heston model calculated using Malliavin weights (M), Likelihood Ratio Method (L), Likelihood Ratio Method combined with SNE or FMR importance sampling schemes and that calculated based on semi-analytical solution. Relative error is measured against the semi-analytical solution. Tables 6 and 7 present results for delta and gamma, respectively, of a European option under the Heston model. Tables 8 and 9 present the results for delta and gamma, respectively, of a barrier option under the Heston model.

It is clear from Table 6 that the Likelihood Ratio Method gives significantly lower relative error than the method of calculating the Greeks using the Malliavin weights. The importance sampling further enhance the efficiency with variance reduction for SNE of up to 23 times and FMR variance reduction of up to 130 times.

The superiority of the Likelihood Ratio Method carries on to the gamma calculation presented in Table 7. This time, the variance reduction for SNE is up to 30 times. For FMR, variance reduction is up to 60 times. 


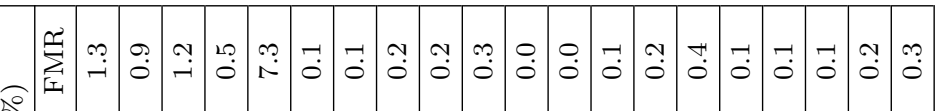

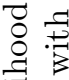

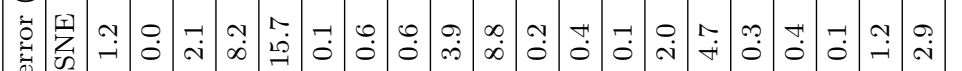

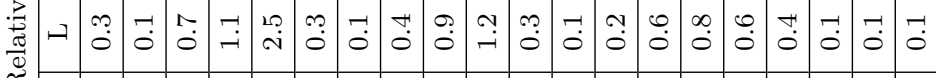

$\approx$

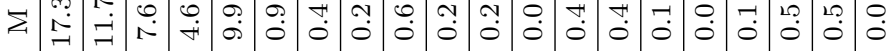

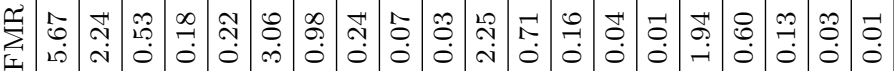

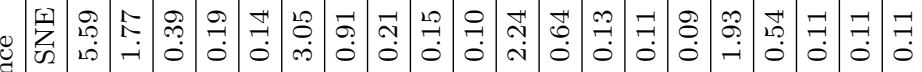

赵

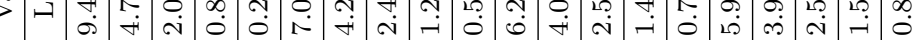

น

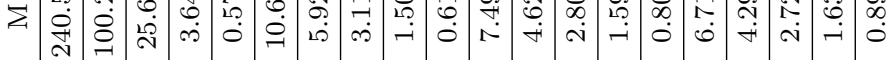

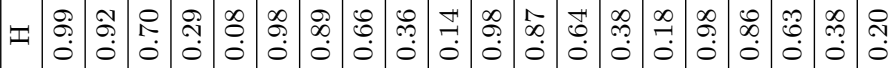

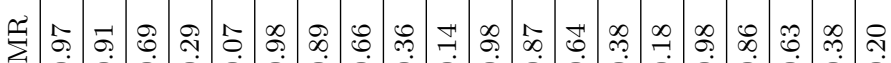

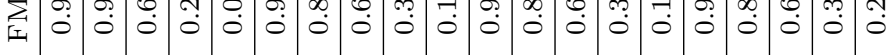

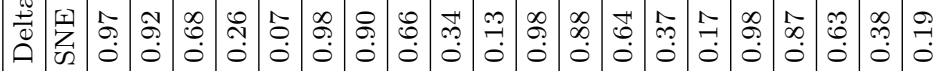

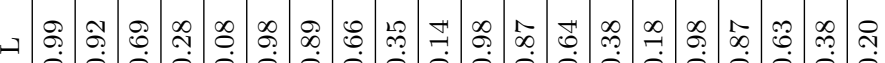

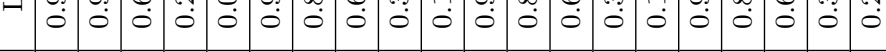

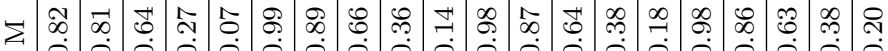

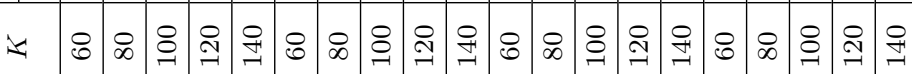

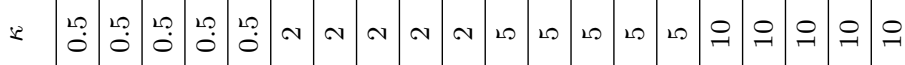

要

تี

国部

过

离苞

奐

.

苋島

สี 해

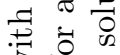
동

帝

ब.

总

"ृ

สี

可

3 .

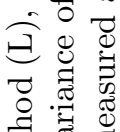

है

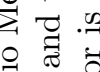

串䨌

엉

용

叫

政

卧

王

吾

零

$\ddot{0} \approx$

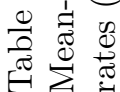




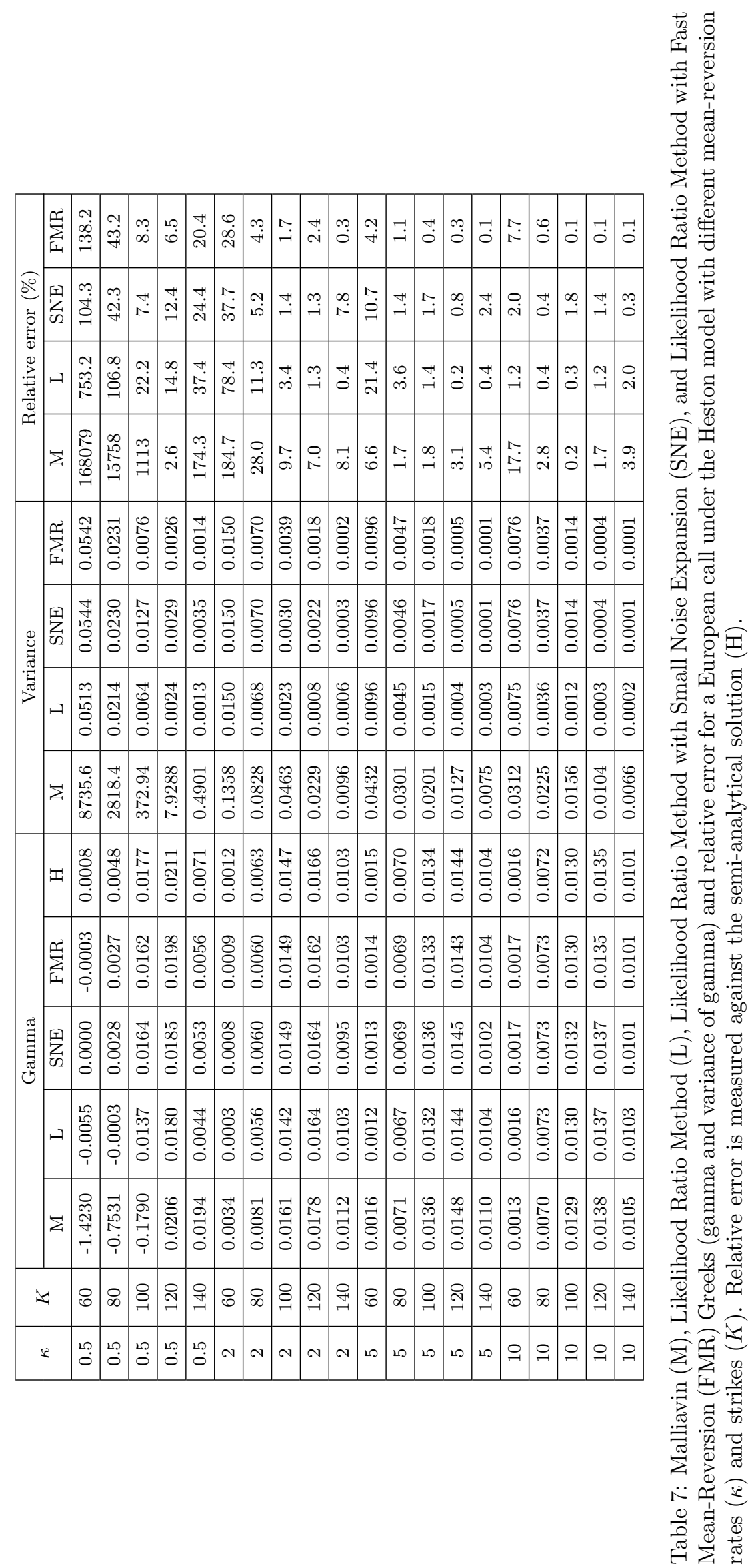




\begin{tabular}{|c|c|c|c|c|c|c|c|c|c|}
\hline \multirow{2}{*}{$\kappa$} & \multirow{2}{*}{$K$} & \multicolumn{5}{|c|}{ Delta } & \multicolumn{4}{c|}{ Variance } \\
\cline { 3 - 10 } & & $\mathrm{M}$ & $\mathrm{L}$ & $\mathrm{SNE}$ & FMR & $\mathrm{M}$ & $\mathrm{L}$ & $\mathrm{SNE}$ & FMR \\
\hline 0.5 & 60 & -0.0030 & -0.0028 & -0.0008 & -0.0017 & 0.0014 & 0.0008 & 0.0001 & 0.0002 \\
\hline 0.5 & 80 & -0.0524 & -0.0522 & -0.0407 & -0.0475 & 0.0847 & 0.0368 & 0.0155 & 0.0109 \\
\hline 0.5 & 100 & -0.2567 & -0.2564 & -0.2428 & -0.2484 & 1.0628 & 0.2308 & 0.0844 & 0.0329 \\
\hline 0.5 & 120 & -0.6672 & -0.6453 & -0.6354 & -0.6366 & 9.3291 & 0.7078 & 0.1776 & 0.1679 \\
\hline 0.5 & 140 & -0.8890 & -0.8340 & -0.8360 & -0.8286 & 40.7111 & 2.1643 & 1.3619 & 1.3908 \\
\hline 2 & 60 & -0.0041 & -0.0044 & -0.0016 & -0.0029 & 0.0017 & 0.0013 & 0.0007 & 0.0001 \\
\hline 2 & 80 & -0.0722 & -0.0741 & -0.0636 & -0.0689 & 0.0808 & 0.0554 & 0.0387 & 0.0043 \\
\hline 2 & 100 & -0.2820 & -0.2815 & -0.2713 & -0.2752 & 0.5015 & 0.2784 & 0.0658 & 0.0222 \\
\hline 2 & 120 & -0.5641 & -0.5597 & -0.5558 & -0.5543 & 1.5981 & 0.7167 & 0.1172 & 0.1183 \\
\hline 2 & 140 & -0.7613 & -0.7513 & -0.7517 & -0.7505 & 3.8517 & 1.5696 & 0.6151 & 0.6255 \\
\hline 5 & 60 & -0.0049 & -0.0050 & -0.0028 & -0.0040 & 0.0020 & 0.0016 & 0.0013 & 0.0000 \\
\hline 5 & 80 & -0.0867 & -0.0883 & -0.0811 & -0.0837 & 0.0856 & 0.0670 & 0.0605 & 0.0025 \\
\hline 5 & 100 & -0.2927 & -0.2929 & -0.2859 & -0.2859 & 0.4428 & 0.3047 & 0.0793 & 0.0183 \\
\hline 5 & 120 & -0.5321 & -0.5311 & -0.5269 & -0.5252 & 1.2288 & 0.7487 & 0.1094 & 0.1253 \\
\hline 5 & 140 & -0.7041 & -0.7023 & -0.6990 & -0.7005 & 2.6741 & 1.5321 & 0.5250 & 0.5635 \\
\hline 10 & 60 & -0.0051 & -0.0054 & -0.0039 & -0.0049 & 0.0019 & 0.0018 & 0.0034 & 0.0000 \\
\hline 10 & 80 & -0.0937 & -0.0955 & -0.0897 & -0.0918 & 0.0853 & 0.0742 & 0.0611 & 0.0018 \\
\hline 10 & 100 & -0.2987 & -0.3005 & -0.2945 & -0.2945 & 0.4153 & 0.3249 & 0.0642 & 0.0195 \\
\hline 10 & 120 & -0.5240 & -0.5259 & -0.5218 & -0.5206 & 1.1042 & 0.7870 & 0.1124 & 0.1435 \\
\hline 10 & 140 & -0.6846 & -0.6871 & -0.6858 & -0.6833 & 2.3388 & 1.5828 & 0.5381 & 0.5937 \\
\hline
\end{tabular}

Table 8: Malliavin (M), Likelihood Ratio Method (L), Likelihood Ratio Method with Small Noise Expansion (SNE), and Likelihood Ratio Method with Fast Mean-Reversion (FMR) Greeks (delta and variance of delta) for a down-and-out put under the Heston model with different mean-reversion rates $(\kappa)$ and strikes $(K)$.

For the delta of a down-and-out put, presented in Table 8, variance reduction for SNE is up to 12 times. For FMR, variance reduction is up to 68 times.

For the gamma of a down-and-out put, presented in Table 9, variance reduction for SNE is up to 6 times. For FMR, variance reduction is up to 70 times.

\subsection{Bates Greeks}

In this section, we present delta, $\Delta$, and gamma, $\Gamma$, of the Bates model calculated using Likelihood Ratio Method (L) and Likelihood Ratio Method combined with SNE or FMR importance sampling schemes. Tables 10 and 11 present results for delta and gamma, respectively, of a European option under the Bates model. Tables 12 and 13 present the results for delta and gamma, respectively, of a barrier option under the Bates model. 


\begin{tabular}{|c|c|c|c|c|c|c|c|c|c|}
\hline \multirow{2}{*}{$\kappa$} & \multirow{2}{*}{$K$} & \multicolumn{4}{|c|}{ Gamma } & \multicolumn{4}{c|}{ Variance } \\
\cline { 3 - 10 } & & $\mathrm{M}$ & $\mathrm{L}$ & $\mathrm{SNE}$ & FMR & $\mathrm{M}$ & $\mathrm{L}$ & $\mathrm{SNE}$ & FMR \\
\hline 0.5 & 60 & 0.0002 & 0.0001 & 0.0000 & 0.0001 & 0.0000 & 0.0000 & 0.0000 & 0.0000 \\
\hline 0.5 & 80 & 0.0031 & 0.0030 & 0.0024 & 0.0028 & 0.0043 & 0.0001 & 0.0001 & 0.0000 \\
\hline 0.5 & 100 & 0.0223 & 0.0146 & 0.0140 & 0.0143 & 0.6736 & 0.0008 & 0.0003 & 0.0001 \\
\hline 0.5 & 120 & -0.0355 & 0.0167 & 0.0173 & 0.0169 & 26.745 & 0.0039 & 0.0023 & 0.0026 \\
\hline 0.5 & 140 & -0.2221 & 0.0015 & 0.0010 & 0.0016 & 189.84 & 0.0141 & 0.0128 & 0.0125 \\
\hline 2 & 60 & 0.0002 & 0.0003 & 0.0001 & 0.0002 & 0.0000 & 0.0000 & 0.0000 & 0.0000 \\
\hline 2 & 80 & 0.0036 & 0.0039 & 0.0034 & 0.0036 & 0.0007 & 0.0002 & 0.0001 & 0.0000 \\
\hline 2 & 100 & 0.0104 & 0.0108 & 0.0105 & 0.0105 & 0.0052 & 0.0008 & 0.0001 & 0.0001 \\
\hline 2 & 120 & 0.0107 & 0.0112 & 0.0114 & 0.0112 & 0.0177 & 0.0023 & 0.0009 & 0.0008 \\
\hline 2 & 140 & 0.0026 & 0.0034 & 0.0034 & 0.0035 & 0.0417 & 0.0052 & 0.0031 & 0.0031 \\
\hline 5 & 60 & 0.0003 & 0.0003 & 0.0002 & 0.0002 & 0.0000 & 0.0000 & 0.0000 & 0.0000 \\
\hline 5 & 80 & 0.0038 & 0.0040 & 0.0038 & 0.0038 & 0.0005 & 0.0002 & 0.0001 & 0.0000 \\
\hline 5 & 100 & 0.0083 & 0.0087 & 0.0086 & 0.0085 & 0.0025 & 0.0008 & 0.0001 & 0.0001 \\
\hline 5 & 120 & 0.0073 & 0.0080 & 0.0079 & 0.0078 & 0.0068 & 0.0020 & 0.0007 & 0.0007 \\
\hline 5 & 140 & 0.0013 & 0.0022 & 0.0020 & 0.0022 & 0.0139 & 0.0041 & 0.0021 & 0.0022 \\
\hline 10 & 60 & 0.0003 & 0.0003 & 0.0002 & 0.0003 & 0.0000 & 0.0000 & 0.0000 & 0.0000 \\
\hline 10 & 80 & 0.0038 & 0.0041 & 0.0039 & 0.0039 & 0.0004 & 0.0002 & 0.0001 & 0.0000 \\
\hline 10 & 100 & 0.0074 & 0.0079 & 0.0079 & 0.0078 & 0.0018 & 0.0008 & 0.0001 & 0.0001 \\
\hline 10 & 120 & 0.0058 & 0.0066 & 0.0066 & 0.0066 & 0.0048 & 0.0020 & 0.0007 & 0.0007 \\
\hline 10 & 140 & 0.0002 & 0.0013 & 0.0012 & 0.0012 & 0.0095 & 0.0040 & 0.0020 & 0.0021 \\
\hline
\end{tabular}

Table 9: Malliavin (M), Likelihood Ratio Method (L), Likelihood Ratio Method with Small Noise Expansion (SNE), and Likelihood Ratio Method with Fast Mean-Reversion (FMR) Greeks (gamma and variance of gamma) for a down-and-out put under the Heston model with different meanreversion rates $(\kappa)$ and strikes $(K)$.

\begin{tabular}{|c|c|c|c|c|c|c|c|c|c|c|c|}
\hline \multirow{2}{*}{$\kappa$} & \multirow{2}{*}{$K$} & \multicolumn{4}{|c|}{ Delta } & \multicolumn{3}{|c|}{ Variance } & \multicolumn{3}{|c|}{ Relative error $(\%)$} \\
\cline { 3 - 13 } & & L & SNE & FMR & B & L & SNE & FMR & L & SNE & FMR \\
\hline 0.5 & 60 & 1.01 & 0.98 & 0.98 & 0.99 & 10.77 & 5.19 & 5.22 & 2.1 & 0.4 & 0.8 \\
\hline 0.5 & 80 & 0.93 & 0.94 & 0.92 & 0.92 & 6.26 & 5.95 & 2.23 & 1.7 & 2.3 & 0.3 \\
\hline 0.5 & 100 & 0.70 & 0.68 & 0.69 & 0.69 & 3.51 & 0.53 & 0.43 & 1.6 & 0.6 & 0.1 \\
\hline 0.5 & 120 & 0.31 & 0.28 & 0.30 & 0.30 & 2.01 & 0.40 & 0.43 & 3.8 & 6.4 & 0.9 \\
\hline 0.5 & 140 & 0.10 & 0.07 & 0.08 & 0.09 & 1.21 & 0.20 & 0.49 & 15.5 & 14.2 & 10.9 \\
\hline 2 & 60 & 0.99 & 0.98 & 0.98 & 0.98 & 7.01 & 3.00 & 3.00 & 0.6 & 0.4 & 0.2 \\
\hline 2 & 80 & 0.89 & 0.89 & 0.89 & 0.89 & 4.26 & 0.95 & 1.02 & 0.3 & 0.7 & 0.2 \\
\hline 2 & 100 & 0.66 & 0.65 & 0.66 & 0.66 & 2.49 & 0.26 & 0.26 & 0.0 & 0.6 & 0.2 \\
\hline 2 & 120 & 0.36 & 0.35 & 0.36 & 0.36 & 1.33 & 0.19 & 0.09 & 0.2 & 4.2 & 0.1 \\
\hline 2 & 140 & 0.15 & 0.14 & 0.15 & 0.15 & 0.61 & 0.12 & 0.03 & 1.1 & 9.8 & 0.2 \\
\hline 5 & 60 & 0.99 & 0.98 & 0.98 & 0.98 & 6.28 & 2.24 & 2.25 & 0.8 & 0.5 & 0.3 \\
\hline 5 & 80 & 0.87 & 0.87 & 0.87 & 0.87 & 4.08 & 0.69 & 0.75 & 0.5 & 0.6 & 0.2 \\
\hline 5 & 100 & 0.64 & 0.64 & 0.64 & 0.64 & 2.60 & 0.17 & 0.20 & 0.3 & 0.2 & 0.2 \\
\hline 5 & 120 & 0.38 & 0.37 & 0.38 & 0.38 & 1.55 & 0.13 & 0.05 & 0.2 & 2.2 & 0.1 \\
\hline 5 & 140 & 0.19 & 0.18 & 0.19 & 0.19 & 0.81 & 0.10 & 0.02 & 0.7 & 6.1 & 0.1 \\
\hline 10 & 60 & 0.99 & 0.98 & 0.98 & 0.98 & 5.93 & 1.95 & 1.95 & 1.1 & 0.5 & 0.4 \\
\hline 10 & 80 & 0.87 & 0.87 & 0.86 & 0.86 & 3.95 & 0.58 & 0.64 & 0.8 & 0.6 & 0.3 \\
\hline 10 & 100 & 0.64 & 0.63 & 0.64 & 0.63 & 2.61 & 0.14 & 0.17 & 0.6 & 0.0 & 0.2 \\
\hline 10 & 120 & 0.39 & 0.38 & 0.39 & 0.39 & 1.62 & 0.12 & 0.04 & 0.5 & 1.7 & 0.2 \\
\hline 10 & 140 & 0.21 & 0.20 & 0.20 & 0.20 & 0.91 & 0.11 & 0.01 & 0.9 & 4.3 & 0.2 \\
\hline
\end{tabular}

Table 10: Likelihood Ratio Method (L), Likelihood Ratio Method with Small Noise Expansion (SNE), and Likelihood Ratio Method with Fast Mean-Reversion (FMR) Greeks (delta and variance of delta) and relative error for a European call under the Bates model with different mean-reversion rates $(\kappa)$ and strikes $(K)$. Relative error is measured against the semi-analytical solution (B). 
焉

\begin{tabular}{|c|c|c|c|c|c|c|c|c|c|c|c|c|c|c|c|c|c|c|c|}
\hline & 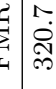 & 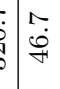 & 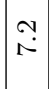 & $\begin{array}{l}0 \\
\infty\end{array}$ & $\overrightarrow{\dot{m}}$ & & $\overrightarrow{+}$ & & & & & & $\stackrel{0}{\circ}$ & $\overrightarrow{0}$ & $\stackrel{0}{\circ}$ & 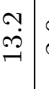 & & & 8 \\
\hline $\begin{array}{l}\frac{y}{x} \\
z \\
y\end{array}$ & $\underset{n}{\vec{n}}$ & & $\stackrel{\leftrightarrow}{\leftrightarrow}$ & 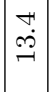 & 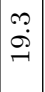 & $\begin{array}{l}0 \\
\dot{\phi} \\
\dot{+}\end{array}$ & $\dot{q}$ & $\underset{\infty}{\infty}$ & $\stackrel{\overbrace{}}{\rightarrow}$ & $\begin{array}{l}\infty \\
\infty \\
\infty\end{array}$ & 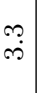 & $\stackrel{m}{g}$ & $\overrightarrow{\mathrm{i}}$ & $\begin{array}{l}0 \\
\stackrel{0}{\circ}\end{array}$ & $\begin{array}{c}\underset{c}{\infty} \\
\infty \\
\infty\end{array}$ & $\vec{\sigma}: \vec{a}$ & & & 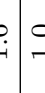 \\
\hline & & & \begin{tabular}{|c|}
$\infty$ \\
$\infty$ \\
$\stackrel{0}{0}$
\end{tabular} & $\overrightarrow{9}$ & $\mid \begin{array}{l}\infty \\
\vec{F} \\
\vec{F}\end{array}$ & 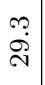 & $\stackrel{\sim}{\leftrightarrow}$ & $\overrightarrow{0}$ & $\stackrel{\overbrace{}}{\rightarrow}$ & $\vec{s}$ & $\stackrel{0}{\stackrel{1}{\oplus}}$ & $\stackrel{\bullet}{\stackrel{1}{\mid}}$ & 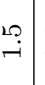 & 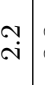 & 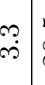 & 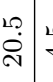 & & & $\because q$ \\
\hline & 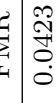 & 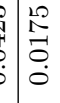 & \begin{tabular}{|l}
2 \\
2 \\
0 \\
0 \\
0 \\
0 \\
0
\end{tabular} & $\left|\begin{array}{l}1 \\
\infty \\
0 \\
0 \\
0\end{array}\right|$ & $\mid \begin{array}{l}m \\
0 \\
0 \\
0 \\
0\end{array}$ & 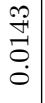 & $\begin{array}{l}\mathscr{g} \\
\stackrel{8}{0} \\
\dot{0}\end{array}$ & 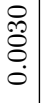 & $\begin{array}{l}\hat{o} \\
\vdots \\
0 \\
0\end{array}$ & $\begin{array}{l}\mathcal{T} \\
\vdots \\
0 \\
0\end{array}$ & 然 & 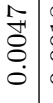 & 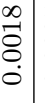 & 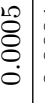 & 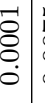 & 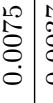 & & & \\
\hline & 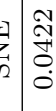 & \begin{tabular}{c|c}
12 \\
$\vec{S}$ \\
0 \\
0 \\
0
\end{tabular} & 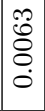 & \begin{tabular}{|l}
$\overrightarrow{0}$ \\
0 \\
0 \\
0 \\
0
\end{tabular} & $\mid \begin{array}{l}0 \\
0 \\
0 \\
0 \\
0\end{array}$ & 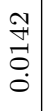 & 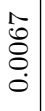 & $\begin{array}{l}\hat{a} \\
0 \\
0 \\
0\end{array}$ & $\begin{array}{l}0 \\
\vdots \\
0 \\
0 \\
0\end{array}$ & $\begin{array}{l}\infty \\
0 \\
0 \\
0\end{array}$ & $\begin{array}{l}1 \\
\stackrel{1}{0} \\
0 \\
0 \\
0\end{array}$ & $\begin{array}{l}\frac{2}{9} \\
0 \\
0 \\
0\end{array}$ & $\begin{array}{l}0 \\
\vdots \\
0 \\
0\end{array}$ & 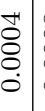 & 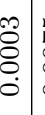 & to & & & \\
\hline & 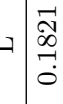 & 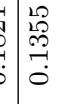 & $\mid \begin{array}{l}\infty \\
8 \\
8 \\
0 \\
0\end{array}$ & $\mid \begin{array}{l}0 \\
\mathbb{N} \\
\hat{N} \\
0 \\
0\end{array}$ & $\begin{array}{l}\hat{0} \\
\hat{0} \\
0 \\
0 \\
0 \\
0\end{array}$ & 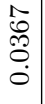 & 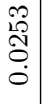 & $\begin{array}{l}0 \\
0 \\
0 \\
0 \\
0\end{array}$ & 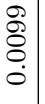 & $\begin{array}{l}0 \\
: \\
0 \\
0 \\
0\end{array}$ & 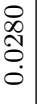 & 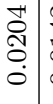 & $\begin{array}{l}\vec{y} \\
\overrightarrow{0} \\
0 \\
0\end{array}$ & $\begin{array}{l}10 \\
8 \\
8 \\
0 \\
0\end{array}$ & 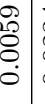 & ָొ & $\begin{array}{c}0 \\
5\end{array}$ & & \\
\hline & m & $\begin{array}{l}17 \\
8 \\
0 \\
0 \\
0\end{array}$ & $\mid \begin{array}{l}\mathbb{N} \\
0 \\
0 \\
0 \\
\end{array}$ & $\mid \begin{array}{l}2 \\
\stackrel{2}{0} \\
\stackrel{0}{0} \\
0\end{array}$ & $\mid \begin{array}{l}\infty \\
0 \\
0 \\
0 \\
0 \\
0\end{array}$ & $\begin{array}{l}m \\
\stackrel{m}{\Xi} \\
\stackrel{0}{\circ}\end{array}$ & $\begin{array}{l}\ddot{\circ} \\
\stackrel{8}{0} \\
\dot{0}\end{array}$ & $\begin{array}{l}\vec{J} \\
\vdots \\
\vdots \\
\dot{0}\end{array}$ & $\begin{array}{l}\mathbb{0} \\
\stackrel{0}{0} \\
\dot{0} \\
0\end{array}$ & $\begin{array}{l}2 \\
0 \\
0 \\
0 \\
0\end{array}$ & $\begin{array}{l}0 \\
\vdots \\
0 \\
0\end{array}$ & $\begin{array}{l}? \\
0 \\
0 \\
\vdots \\
0\end{array}$ & $\begin{array}{l}\overrightarrow{0} \\
\overrightarrow{0} \\
\dot{0} \\
\end{array}$ & $\begin{array}{c}\vec{F} \\
\vdots \\
0 \\
0\end{array}$ & $\begin{array}{l}\overrightarrow{0} \\
0 \\
0\end{array}$ & & & & \\
\hline & I & 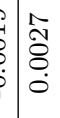 & $\mid \begin{array}{l}0 \\
0 \\
0 \\
0 \\
0\end{array}$ & $\mid \begin{array}{l}\infty \\
\infty \\
0 \\
0 \\
0\end{array}$ & $\mid \begin{array}{l}0 \\
0 \\
0 \\
0 \\
0 \\
0\end{array}$ & 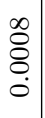 & $\begin{array}{l}\overrightarrow{0} \\
\dot{\Xi} \\
\dot{0}\end{array}$ & 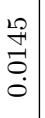 & $\begin{array}{l}\text { त̂ } \\
0 \\
0 \\
0\end{array}$ & $\begin{array}{l}\overrightarrow{0} \\
\overrightarrow{0} \\
\dot{0}\end{array}$ & $\begin{array}{l}0 \\
\vdots \\
0 \\
0 \\
0\end{array}$ & 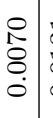 & $\begin{array}{l}\overrightarrow{0} \\
\overrightarrow{0} \\
\dot{0} \\
\dot{0}\end{array}$ & $\begin{array}{c}\vec{F} \\
\overrightarrow{0} \\
0 \\
0\end{array}$ & $\begin{array}{l}\overrightarrow{0} \\
0 \\
0\end{array}$ & $\overrightarrow{8}$ & & 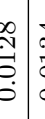 & \\
\hline & $\sum_{2}$ & 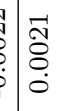 & $\mid \begin{array}{l}\infty \\
0 \\
0 \\
0 \\
0\end{array}$ & $\left|\begin{array}{l}\infty \\
0 \\
0 \\
0 \\
0\end{array}\right|$ & $\mid \begin{array}{l}\mathscr{0} \\
0 \\
0 \\
0 \\
0\end{array}$ & $\begin{array}{l}\hat{0} \\
0 \\
0 \\
0\end{array}$ & $\begin{array}{l}\overrightarrow{0} \\
\dot{0} \\
\dot{0}\end{array}$ & $\begin{array}{l}\text { 靑 } \\
\vdots \\
0 \\
0\end{array}$ & $\begin{array}{l}0 \\
0 \\
0 \\
0 \\
0\end{array}$ & $\begin{array}{l}0 \\
0 \\
0 \\
0\end{array}$ & 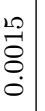 & $\begin{array}{l}0 \\
0 \\
0 \\
0 \\
0\end{array}$ & $\begin{array}{l}\frac{1}{0} \\
0 \\
0 \\
0\end{array}$ & $\begin{array}{c}\mathcal{I} \\
0 \\
0 \\
0\end{array}$ & $\begin{array}{l}\overrightarrow{0} \\
0 \\
0 \\
0\end{array}$ & & & & \\
\hline & $\begin{array}{l}0 \\
\stackrel{8}{0} \\
\dot{0}\end{array}$ & 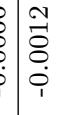 & 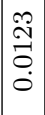 & & 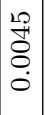 & $\begin{array}{l}0 \\
\dot{o} \\
0 \\
\dot{0}\end{array}$ & $\begin{array}{l}1 \\
\stackrel{8}{0} \\
\dot{0}\end{array}$ & $\begin{array}{l}\stackrel{⿰}{\rightarrow} \\
\stackrel{⿰}{0} \\
\dot{0}\end{array}$ & $\begin{array}{l}\vec{J} \\
\stackrel{0}{0} \\
0 \\
0\end{array}$ & $\begin{array}{l}\infty \\
0 \\
\vdots \\
0 \\
0\end{array}$ & 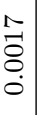 & $\begin{array}{l}\overrightarrow{5} \\
8 \\
0 \\
\dot{0}\end{array}$ & $\begin{array}{l}0 \\
0 \\
0 \\
0 \\
0\end{array}$ & 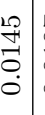 & & & & & \\
\hline$\approx$ & 8 & $\infty \infty$ & 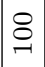 & 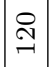 & $\stackrel{ }{+}$ & 8 & $\triangleright$ & 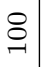 & ঙิ & $\underset{\exists}{\rightarrow}$ & 8 & $\triangleright$ & $\stackrel{8}{.}$ & 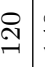 & I & 0 & $\infty$ & 更 & \\
\hline & 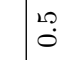 & \begin{tabular}{l|l}
$\dot{L}$ \\
$\dot{0}$
\end{tabular} & & & & & & & N & N & 10 & 10 & & & & . & o & & \\
\hline
\end{tabular}

䞸

空

$\circ$

을

ज्ञ

ซี

玨昰

觉晜

울

क्षै

वे

暐

궁도

ซี

क्ष

견엉.

5

을

荧

윰

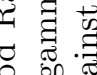

$00 \%$

过

宅芯

뭉.

荧

串.

党

过

$\rightarrow \widetilde{\exists}$

密运象

$\ddot{-}$ 。

ㄱ.

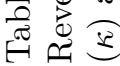




\begin{tabular}{|c|c|c|c|c|c|c|c|}
\hline \multirow{2}{*}{$\kappa$} & \multirow{2}{*}{$K$} & \multicolumn{3}{|c|}{ Delta } & \multicolumn{3}{|c|}{ Variance } \\
\cline { 3 - 8 } & & $\mathrm{L}$ & SNE & FMR & L & SNE & FMR \\
\hline 0.5 & 60 & -0.0027 & -0.0008 & -0.0018 & 0.0008 & 0.0001 & 0.0002 \\
\hline 0.5 & 80 & -0.0537 & -0.0429 & -0.0490 & 0.0375 & 0.0196 & 0.0111 \\
\hline 0.5 & 100 & -0.2595 & -0.2462 & -0.2525 & 0.2347 & 0.0910 & 0.0430 \\
\hline 0.5 & 120 & -0.6204 & -0.6140 & -0.6149 & 0.7015 & 0.1981 & 0.1823 \\
\hline 0.5 & 140 & -0.8126 & -0.8174 & -0.8113 & 1.9645 & 1.1706 & 1.2266 \\
\hline 2 & 60 & -0.0040 & -0.0015 & -0.0029 & 0.0012 & 0.0004 & 0.0001 \\
\hline 2 & 80 & -0.0735 & -0.0640 & -0.0693 & 0.0532 & 0.0431 & 0.0055 \\
\hline 2 & 100 & -0.2763 & -0.2682 & -0.2716 & 0.2683 & 0.0927 & 0.0284 \\
\hline 2 & 120 & -0.5428 & -0.5411 & -0.5395 & 0.6893 & 0.1322 & 0.1322 \\
\hline 2 & 140 & -0.7281 & -0.7324 & -0.7296 & 1.4952 & 0.6150 & 0.6209 \\
\hline 5 & 60 & -0.0049 & -0.0025 & -0.0041 & 0.0016 & 0.0008 & 0.0000 \\
\hline 5 & 80 & -0.0869 & -0.0788 & -0.0830 & 0.0640 & 0.0573 & 0.0038 \\
\hline 5 & 100 & -0.2852 & -0.2785 & -0.2795 & 0.2901 & 0.0771 & 0.0235 \\
\hline 5 & 120 & -0.5145 & -0.5113 & -0.5108 & 0.7113 & 0.1208 & 0.1334 \\
\hline 5 & 140 & -0.6794 & -0.6805 & -0.6798 & 1.4511 & 0.5210 & 0.5536 \\
\hline 10 & 60 & -0.0052 & -0.0035 & -0.0048 & 0.0017 & 0.0021 & 0.0000 \\
\hline 10 & 80 & -0.0937 & -0.0875 & -0.0901 & 0.0701 & 0.0634 & 0.0030 \\
\hline 10 & 100 & -0.2922 & -0.2856 & -0.2870 & 0.3068 & 0.0659 & 0.0245 \\
\hline 10 & 120 & -0.5097 & -0.5071 & -0.5053 & 0.7425 & 0.1240 & 0.1478 \\
\hline 10 & 140 & -0.6653 & -0.6660 & -0.6632 & 1.4913 & 0.5268 & 0.5776 \\
\hline
\end{tabular}

Table 12: Likelihood Ratio Method (L), Likelihood Ratio Method with Small Noise Expansion (SNE), and Likelihood Ratio Method with Fast Mean-Reversion (FMR) Greeks (delta and variance of delta) for a down-and-out put under the Bates model with different mean-reversion rates $(\kappa)$ and strikes $(K)$.

For the delta of European call, presented in Table 10, variance reduction of SNE is up to 19 times. For FMR, variance reduction is up to 76 times. For the gamma of European call, presented in Table 11, SNE gives up to 27 times variance reduction. For FMR, variance reduction is up to 50 times.

For the delta of a down-and-out put, presented in Table 12, SNE reduces variance up to 12 times, while FMR gives variance reduction of up to 44 times. For the gamma of a down-and-out put, presented in Table 13, SNE reduces variance up to 10 times, while FMR gives variance reduction of up to 46 times.

\section{Conclusion}

In this paper we provide strong evidence that importance sampling reduces the variance of European and barrier options and in most cases it clearly outperforms the control variate. We have 


\begin{tabular}{|c|c|c|c|c|c|c|c|}
\hline \multirow{2}{*}{$\kappa$} & \multirow{2}{*}{$K$} & \multicolumn{3}{|c|}{ Gamma } & \multicolumn{3}{c|}{ Variance } \\
\cline { 3 - 8 } & & $\mathrm{L}$ & SNE & FMR & L & SNE & FMR \\
\hline 0.5 & 60 & 0.0001 & 0.0000 & 0.0001 & 0.0000 & 0.0000 & 0.0000 \\
\hline 0.5 & 80 & 0.0032 & 0.0025 & 0.0029 & 0.0001 & 0.0001 & 0.0000 \\
\hline 0.5 & 100 & 0.0140 & 0.0134 & 0.0136 & 0.0008 & 0.0003 & 0.0001 \\
\hline 0.5 & 120 & 0.0157 & 0.0162 & 0.0159 & 0.0033 & 0.0019 & 0.0018 \\
\hline 0.5 & 140 & 0.0013 & 0.0013 & 0.0016 & 0.0107 & 0.0091 & 0.0089 \\
\hline 2 & 60 & 0.0002 & 0.0001 & 0.0002 & 0.0000 & 0.0000 & 0.0000 \\
\hline 2 & 80 & 0.0038 & 0.0033 & 0.0035 & 0.0002 & 0.0001 & 0.0000 \\
\hline 2 & 100 & 0.0101 & 0.0099 & 0.0099 & 0.0007 & 0.0002 & 0.0001 \\
\hline 2 & 120 & 0.0102 & 0.0105 & 0.0103 & 0.0021 & 0.0008 & 0.0008 \\
\hline 2 & 140 & 0.0027 & 0.0030 & 0.0029 & 0.0047 & 0.0028 & 0.0028 \\
\hline 5 & 60 & 0.0003 & 0.0001 & 0.0002 & 0.0000 & 0.0000 & 0.0000 \\
\hline 5 & 80 & 0.0038 & 0.0035 & 0.0037 & 0.0002 & 0.0001 & 0.0000 \\
\hline 5 & 100 & 0.0081 & 0.0080 & 0.0079 & 0.0007 & 0.0001 & 0.0001 \\
\hline 5 & 120 & 0.0071 & 0.0072 & 0.0071 & 0.0018 & 0.0007 & 0.0007 \\
\hline 5 & 140 & 0.0014 & 0.0014 & 0.0014 & 0.0037 & 0.0019 & 0.0020 \\
\hline 10 & 60 & 0.0003 & 0.0002 & 0.0003 & 0.0000 & 0.0000 & 0.0000 \\
\hline 10 & 80 & 0.0038 & 0.0037 & 0.0037 & 0.0002 & 0.0001 & 0.0000 \\
\hline 10 & 100 & 0.0073 & 0.0073 & 0.0072 & 0.0007 & 0.0001 & 0.0001 \\
\hline 10 & 120 & 0.0058 & 0.0060 & 0.0058 & 0.0017 & 0.0007 & 0.0007 \\
\hline 10 & 140 & 0.0005 & 0.0005 & 0.0005 & 0.0035 & 0.0018 & 0.0019 \\
\hline
\end{tabular}

Table 13: Likelihood Ratio Method (L), Likelihood Ratio Method with Small Noise Expansion (SNE), and Likelihood Ratio Method with Fast Mean-Reversion (FMR) Greeks (gamma and variance of gamma) for a down-and-out put under the Bates model with different mean-reversion rates $(\kappa)$ and strikes $(K)$.

also introduced an effective numerical scheme that dramatically improves the speed of both Small Noise Expansion (SNE), Fast Mean-Reversion (FMR) importance sampling schemes. With an appropriately designed lookup table both SNE and FMR outperform the control variate, not only in terms of variance reduction, but also on speed. Finally, we have shown that Likelihood Ratio Method is an efficient way of obtaining the Greeks, and combining it with importance sampling leads to a significant variance reduction for the Greeks. 


\section{References}

[1] Arouna, B. (2004) Robbins-Monro Algorithms and Variance Reduction in Finance. Journal of Computational Finance, 7(2):35-62.

[2] Bates D. (1996) Jumps and Stochastic Volatility: The Exchange Rate Processes Implicit in Deutschemark Options. Review of Financial Studies, 9(1):69-107.

[3] Benhamou, E. (2002) Smart Monte Carlo: Various Tricks Using Malliavin Calculus. Quantitative Finance, 2(5):329-336.

[4] Broadie, M. and Ö. Kaya (2006) Exact Simulation of Option Greeks under Stochastic Volatility and Jump Diffusion Models. Proceedings of the 2004 Winter Simulation Conference, pp. 1607-1615.

[5] Capriotti, L. (2007) Least Squares Importance Sampling for Libor Market Models. Wilmott, Nov 2007.

[6] Capriotti, L. (2008) Least Squares Importance Sampling for Monte Carlo Security Pricing. Quantitative Finance, 8(5):485-497.

[7] Chan, J.C.C. and D.P. Kroese (2010) Efficient Estimation of Large Portfolio Loss Probabilities in t-copula Models. European Journal of Operational Research, 205(2):361-367.

[8] Chen, Z. and P. Glasserman (2008) Fast Pricing of Basket Default Swaps. Operations Research, 56(2):286-303.

[9] Étoré, P. and B. Jourdain (2010) Adaptive Optimal Allocation in Stratified Sampling Methods. Methodology and Computing in Applied Probability, 12(3):335-360.

[10] Fouque, J.P. and C.H. Han (2004) Variance Reduction for Monte Carlo Methods to Evaluate Option Prices under Multi-factor Stochastic Volatility Models. Quantitative Finance, 4(5):597-606.

[11] Fouque, J.P., G. Papanicolaou and K.R. Sircar (2000) Derivatives in Financial Markets with Stochastic Volatility. Cambridge University Press.

[12] Fouque, J.P., G. Papanicolaou and K.R. Sircar (2011) Multiscale Stochastic Volatility for Equity, Interest Rate, and Credit Derivatives. Cambridge University Press. 
[13] Fouque, J.P. and T. Tullie (2002) Variance Reduction for Monte Carlo Simulation in a Stochastic Volatility Environment. Quantitative Finance, 2(1):24-30.

[14] Fournie, E. , J. Lebuchoux and N. Touzi. (1997) Small Noise Expansion and Importance Sampling. Asymptotic Analysis, 14(4):361-376.

[15] Glasserman, P. (2005) Measuring Marginal Risk Contributions in Credit Portfolios. Journal of Computational Finance, 9(2):1-41.

[16] Glasserman, P., P. Heidelberger and P. Shahabuddin (1999a) Asymptotically Optimal Importance Sampling and Stratification for Pricing Path-dependent Options. Mathematical Finance, 9(2):117-152.

[17] Glasserman, P., P. Heidelberger and P. Shahabuddin (1999b) Importance Sampling in the Heath-Jarrow-Morton Framework. Journal of Derivatives, 7(1):32-50.

[18] Glasserman, P. and J. Li (2005) Importance Sampling for Portfolio Credit Risk. Management Science, 51(11):1643-1656.

[19] Glasserman, P. and Z. Liu (2010) Sensitivity Estimates from Characteristic Functions. Operations Research, 58(6):1611-1623.

[20] Glasserman, P., W. Kang and P. Shahabuddin (2008) Fast Simulation of Multifactor Portfolio Credit Risk. Operations Research, 56(5):1200-1217.

[21] Glasserman, P. and J. Staum (2001) Conditioning on One-Step Survival for Barrier Option Simulations. Operations Research, 49(6):923-937.

[22] Grundke, P. (2009) Importance Sampling for Integrated Market and Credit Portfolio Models. European Journal of Operational Research 194(1):206226.

[23] Guasoni, P. and S. Robertson (2008) Optimal Importance Sampling with Explicit Formulas in Continuous Time. Finance and Stochastics, 12(1):1-19.

[24] Haug, E.G. (2006) The Complete Guide to Option Pricing Formulas, 2nd Edition. McGraw-Hill, New York, NY.

[25] Heston H.L. (1993) A Closed-form Solution for Options with Stochastic Volatility with Applications to Bond and Currency Options. Review of Financial Studies, 6(2):327343. 
[26] Huang, P., D. Subramanian, and J. Xu (2010) An Importance Sampling Method for Portfolio CVaR Estimation with Gaussian Copula Models. Proceedings of the 2010 Winter Simulation Conference, pp. 2790-2800.

[27] Joshi, M.S. (2005) Applying Importance Sampling to Pricing Single Tranches of CDOs in a One-Factor Li Model. Wilmott, Mar 2005.

[28] Joshi, M.S. and D.S. Kainth (2004) Rapid Computation of Prices and Greeks for Nth to Default Swaps in the Li Model. Quantitative Finance, 4(3):266-275.

[29] Joshi, M.S. and T.S. Leung (2007) Using Monte Carlo Simulation and Importance Sampling to Rapidly Obtain Jump-Diffusion Prices of Continuous Barrier Options. Journal of Computational Finance, 10(4):93-105.

[30] Kang, W. and P. Shahabuddin (2005) Fast Simulation for Multifactor Portfolio Credit Risk in the t-Copula Model. Proceedings of the 2005 Winter Simulation Conference, pp. 1859-1868.

[31] Merino, S. and M. Nyfeler (2004) Applying Importance Sampling for Estimating Coherent Credit Risk Contributions. Quantitative Finance, 4(2):199-207.

[32] Robertson, S. (2010) Sample Path Large Deviations and Optimal Importance Sampling for Stochastic Volatility Models. Stochastic Processes and their Applications, 120(1):66-83.

[33] Sak, H. and W. Hörmann (2012) Fast Simulations in Credit Risk. Quantitative Finance, 12(10):1557-1569.

[34] Su, Y. and M.C. Fu (2002) Optimal Importance Sampling in Securities Pricing. Journal of Computational Finance, 5(4):27-50.

[35] Tullie, T.A. (2002) Variance Reduction for Monte Carlo Simulation of European, American or Barrier Options in a Stochastic Volatility Environment. PhD thesis. North Carolina State University, Raleigh, NC.

[36] Vázquez-Abad, F.J. and D. Dufresne (1998) Accelerated Simulation for Pricing Asian Options. Proceedings of the 2005 Winter Simulation Conference, pp. 1493-1500. 